\title{
Molecular Mechanisms Controlling Vascular Lumen Formation in Three-Dimensional Extracellular Matrices
}

\author{
Anastasia Sacharidou $^{a} \quad$ Amber N. Stratman ${ }^{a}$ George E. Davis ${ }^{a, b}$ \\ Departments of ${ }^{\mathrm{a}}$ Medical Pharmacology and Physiology and ${ }^{\mathrm{b}}$ Pathology and Anatomical Sciences, \\ University of Missouri School of Medicine, Dalton Cardiovascular Research Center, Columbia, Mo., USA
}

\section{Key Words}

Endothelial lumen formation - Tubulogenesis • Lumen signaling complexes $\cdot$ Cdc42 $\cdot$ Rac1 $\cdot$ MT1-MMP $\cdot$ JamB • JamC $\cdot$ Par3 - Par6b - Pericytes - Vascular guidance tunnels . Vascular basement membrane matrix assembly

\begin{abstract}
Considerable progress has been made toward a molecular understanding of how cells form lumen and tube structures in three-dimensional (3D) extracellular matrices (ECM). This progress has occurred through work performed with endothelial and epithelial cell models using both in vitro and in vivo approaches. Despite the apparent similarities between endothelial and epithelial cell lumen and tube formation mechanisms, there are clear distinctions that directly relate to their functional differences. This review will focus on endothelial cell (EC) lumen formation mechanisms which control blood vessel formation during development and postnatal life. Of great interest is that an EC lumen signaling complex has been identified which controls human EC lumen and tube formation in 3D matrices and which coordinates integrin-ECM contacts, cell surface proteolysis, cytoskeletal rearrangements, and cell polarity. This complex consists of the collagen-binding integrin $\alpha 2 \beta 1$, the collagen-degrading membrane-type 1 matrix metalloproteinase (MT1-MMP),
\end{abstract}

junction adhesion molecule (Jam)C, JamB, polarity proteins Par3 and Par6b, and the Rho GTPase Cdc42-GTP. These interacting proteins are necessary to stimulate 3D matrix-specific signaling events (including activation of protein kinase cascades that regulate the actin and microtubule cytoskeletons) to control the formation of EC lumens and tube networks. Also, EC lumen formation is directly coupled to the

\section{Abbreviations used in this paper}

3D three-dimensional

aPKC atypical protein kinase $\mathrm{C}$

CCM cerebral cavernous malformation

EC endothelial cell

ECM extracellular matrix

GEFs guanine exchange factors

GFP green fluorescent protein

IL-3 interleukin-3

Jam junction adhesion molecule

mRFP monomeric red fluorescent protein

Pak p21-activated kinase

PKC protein kinase $\mathrm{C}$

SCF stem cell factor

SDF stromal-derived factor

STKs sterile 20 kinases

TIMP tissue inhibitor of metalloproteinase

\section{KARGER}

() 2011 S. Karger AG, Basel

Fax +4161306 1234

E-Mail karger@karger.ch

www.karger.com
Accessible online at: www.karger.com/cto
Prof. George E. Davis

Department of Medical Pharmacology and Physiology

University of Missouri School of Medicine, MA415 Medical Sciences Building Columbia, MO 65212 (USA)

Tel. +1 573882 5474, E-Mail davisgeo@ missouri.edu 
generation of vascular guidance tunnels, enzymatically generated ECM conduits that facilitate EC tube remodeling and maturation. Mural cells such as pericytes are recruited along EC tubes within these tunnel spaces to control ECM remodeling events resulting in vascular basement membrane matrix assembly, a key step in tube maturation and stabilization.

Copyright $\odot 2011$ S. Karger AG, Basel

\section{Introduction}

A fundamental required step in vascular morphogenesis is the creation of networks of endothelial cell (EC)lined tubes in both developmental and postnatal contexts [Adams and Alitalo, 2007; Davis et al., 2007, 2011b; Holderfield and Hughes, 2008; Iruela-Arispe and Davis, 2009; Warren and Iruela-Arispe, 2010; Senger and Davis, 2011]. The molecular control of this process is being elucidated using a variety of experimental approaches including both in vitro and in vivo models that have led to major advances in our developing mechanistic understanding of this process. ECs, like epithelial cells, are capable of forming cell-lined tubes when placed in a three-dimensional (3D) extracellular matrix (ECM) environment when appropriate cytokines are supplied (that support morphogenesis and survival) [Iruela-Arispe and Davis, 2009; Andrew and Ewald, 2010; Datta et al., 2011; Davis et al., 2011b; Senger and Davis, 2011]. This is in marked contrast to other cell types such as fibroblasts, pericytes, and vascular smooth muscle cells which fail to form lumens and tubes when they are placed under the same conditions. The molecular basis for this fundamental difference remains a critical question for understanding why ECs and epithelial cells form lumens and tubes and why fibroblasts and other cell types do not.

EC-lined tubes are unique in that they become exposed to high flow and pressure forces [Lucitti et al., 2007; Dejana et al., 2009; Iruela-Arispe and Davis, 2009; Wagenseil and Mecham, 2009; Culver and Dickinson, 2010]. This major functional difference between these tubes and epithelial cell tubes needs to be emphasized. In general, blood EC tubes (contrasting them with lymphatic EC tubes) propel blood cells and plasma fluids and participate in a closed, pressurized circuit connected to a heart pump [Wagenseil and Mecham, 2009; Culver and Dickinson, 2010], while many epithelial tubes are typically secretory in nature but can also propel fluids, albeit in a low pressure environment. These distinctions are highly relevant, and although EC and epithelial tubulogenesis mechanisms are related, their functional dissimilarities likely play major roles in why their tube formation mechanisms show significant differences as well.

Another key distinction is the major accumulation of mural cells that become invested around EC-lined tubes in the blood vasculature [Armulik et al., 2005; Hughes, 2008; Gaengel et al., 2009; Davis et al., 2011a; Senger and Davis, 2011]. This mural cell recruitment process and the functional consequences of these events lead to tubes that are able to control systemic processes such as blood pressure (through arteriolar contractility) and elastic recoil forces (i.e. from large arteries) that propel blood through the vasculature in conjunction with the cardiac contractility cycle [Segal, 2005; Hill et al., 2007; Wagenseil and Mecham, 2009]. The process by which mural cells become recruited to EC-lined tubes is being elucidated using both in vitro and in vivo models [Davis et al., 2007, 2011a, b; Hughes, 2008; Gaengel et al., 2009; Stratman et al., 2009a, 2010]. Of great interest is that pericyte recruitment to EC tubes in the developing and stable microvasculature leads to ECM remodeling and, specifically, vascular basement membrane matrix assembly, a fundamental step in the maturation of the vasculature [Stratman et al., 2009a, 2010].

One apparent distinction between EC and epithelial tubes is the relative lack of transmembrane proteins that are specific to the apical membrane in ECs versus epithelial tubes [Davis et al., 2011b]. Podocalyxin is a transmembrane protein that appears to show apical targeting in ECs [Dekan et al., 1990; Lampugnani et al., 2010]; however, it again appears less than that observed in epithelial tubes. An interesting consideration is that the accumulation of mural cells specifically on the basal surface of EC tubes along with vascular basement membrane at this surface could represent a major polarizing signal for ECs. Importantly, the apical surface in ECs is exposed to significant flow and pressure forces [Culver and Dickinson, 2010] and this type of stimulus may also represent a major polarizing signal for ECs. Flow forces are known to cause centrosome reorientation in the direction of flow, an event that requires Cdc42 [Tzima et al., 2003]. Perhaps the specialized apical surfaces of epithelial cells [Bryant and Mostov, 2008], which are highly linked to secretory and absorptive functions, are not compatible with such forces. ECs are also very thin and flat cells in most vascular beds with small EC-EC junctions compared with the large cuboidal epithelial cell shape and large membrane contact zones in typical epithelial cell-cell junctions [Lampugnani and Dejana, 2007; Bryant and Mostov, 2008; Dejana et al., 2009; Iruela-Arispe and Davis, 2009]. These marked distinctions likely underlie many of 
the new differences that are becoming apparent in studies of both EC and epithelial lumen formation. However, it is also clear that studies of both systems have lead to important advances in our understanding of the lumen and tube formation process that have applicability toward both events.

In this review, the molecular basis for EC lumen and tube morphogenesis will be discussed, and we will emphasize the critical importance of studies using both in vitro and in vivo approaches to elucidate the molecular basis for these fundamental biological processes. We will focus on a number of key issues including the role of ECM, integrin and Rho GTPase signaling, protein kinase cascades, cell polarity mechanisms, multiprotein complexes that control EC lumen formation and signaling, EC-mural cell interactions that influence EC tube formation and vascular basement membrane matrix assembly, and regulation of vascular tube morphogenesis by cytokines.

\section{Morphologic Changes That Characterize the EC Lumen and Tube Assembly Process in 3D ECM: Role of Intracellular Vacuoles in EC Lumen Formation}

It has been important over the years to characterize the EC lumen and tube formation process from a morphologic perspective [Folkman and Haudenschild, 1980; Davis and Camarillo, 1996; Davis et al., 2002; Davis and Bayless, 2003; Lubarsky and Krasnow, 2003; Filla et al., 2004; Sainson et al., 2005; Kamei et al., 2006; Saunders et al., 2006; Yaniv et al., 2006; Zamir et al., 2006; Aplin et al., 2008; Holderfield and Hughes, 2008; Koh et al., 2008b; McKinney and Weinstein, 2008; Nakatsu and Hughes, 2008; Herbert et al., 2009; Sato et al., 2010]. The development of real-time imaging approaches to visualize these events in vitro and in vivo have strongly facilitated our ability to identify and characterize particular steps in the process. In addition, basic histologic approaches have revealed considerable information as well. As shown in figure 1, human ECs are able to assemble into multicellular tube networks when they are suspended as single cells in 3D matrices in assay systems that mimic 'vasculogenesis' or in others where ECs are seeded on the surface of 3D ECM gels and allowed to sprout into the matrix to form tubes and tube networks (mimicking angiogenesis). A key point is that cross sections of such cultures need to demonstrate the presence of lumens as shown in figure $1 c, d$, and $\mathrm{f}$. It is clear that the structures are multicellular (fig. 1a, e) but, to demonstrate this point further, ECs car- rying either green fluorescent protein (GFP) or separately monomeric red fluorescent protein (mRFP) were cocultured to demonstrate multicellular tube assembly during this process (fig. 1b).

Careful imaging of the EC tubulogenic process in 3D matrices in vitro revealed the surprising finding that ECs undergo marked intracellular vacuolation and fusion events during tube formation (fig. 2) [Davis and Camarillo, 1996; Bayless et al., 2000; Bayless and Davis, 2002; Davis et al., 2002; Davis and Bayless, 2003; Kamei et al., 2006]. Importantly, these phenomena have been observed both in vitro and in vivo [Egginton and Gerritsen, 2003; Kamei et al., 2006; Liu et al., 2010; Wang et al., 2010; Zovein et al., 2010]. A critical question that has yet to be answered after all of these years is: why is this necessary? In support of such a concept is the clear observation that single ECs, without contact from neighboring ECs (and without EC-EC junctional contacts), can form lumen structures [Davis and Camarillo, 1996; Davis et al., 2002]. A real-time image series of a single EC forming vacuoles and a lumen structure over $24 \mathrm{~h}$ is shown in figure $2 \mathrm{a}$. Real-time imaging at lower power reveals fields of ECs forming intracellular vacuoles and then expanding their lumens in conjunction with EC sprouting and branching events leading to multicellular tube assembly (fig. 2b). To partially address the question raised above, it is apparent that ECs need to assemble multicellular tube structures very rapidly (within hours) in order to accommodate embryonic developmental requirements (i.e. oxygenation to meet rapid growth needs). Interestingly, EC tubulogenesis in vitro (1-2 days) [Koh et al., 2008b] appears to be much more rapid than that observed for epithelial tubulogenesis (1-2 weeks) [O'Brien et al., 2006]. In order to transfer basal membranes to an apical position rapidly to accommodate the timing of the $\mathrm{EC}$ tubulogenic process, it would make sense to rapidly pinocytose basal membranes into a presumptive apical membrane position (i.e. a process that resembles macropinocytosis) through intracellular vacuole formation and vacuole-vacuole fusion events (fig. 3). In fact, our laboratory has clearly observed that this process occurs during EC lumen formation in vitro [Davis and Camarillo, 1996; Bayless et al., 2000; Davis et al., 2002]. Interestingly, other intracellular membranes appear to fuse with intracellular vacuoles also as we observed that Weibel-Palade bodies could be shown to fuse and transfer their contents (i.e. von Willebrand factor) into early vacuoles and early lumenal structures [Davis and Camarillo, 1996; Davis and Bayless, 2003]. Thus, it appears that ECs may use such mechanisms to rapidly create apical membranes that are fluid filled 

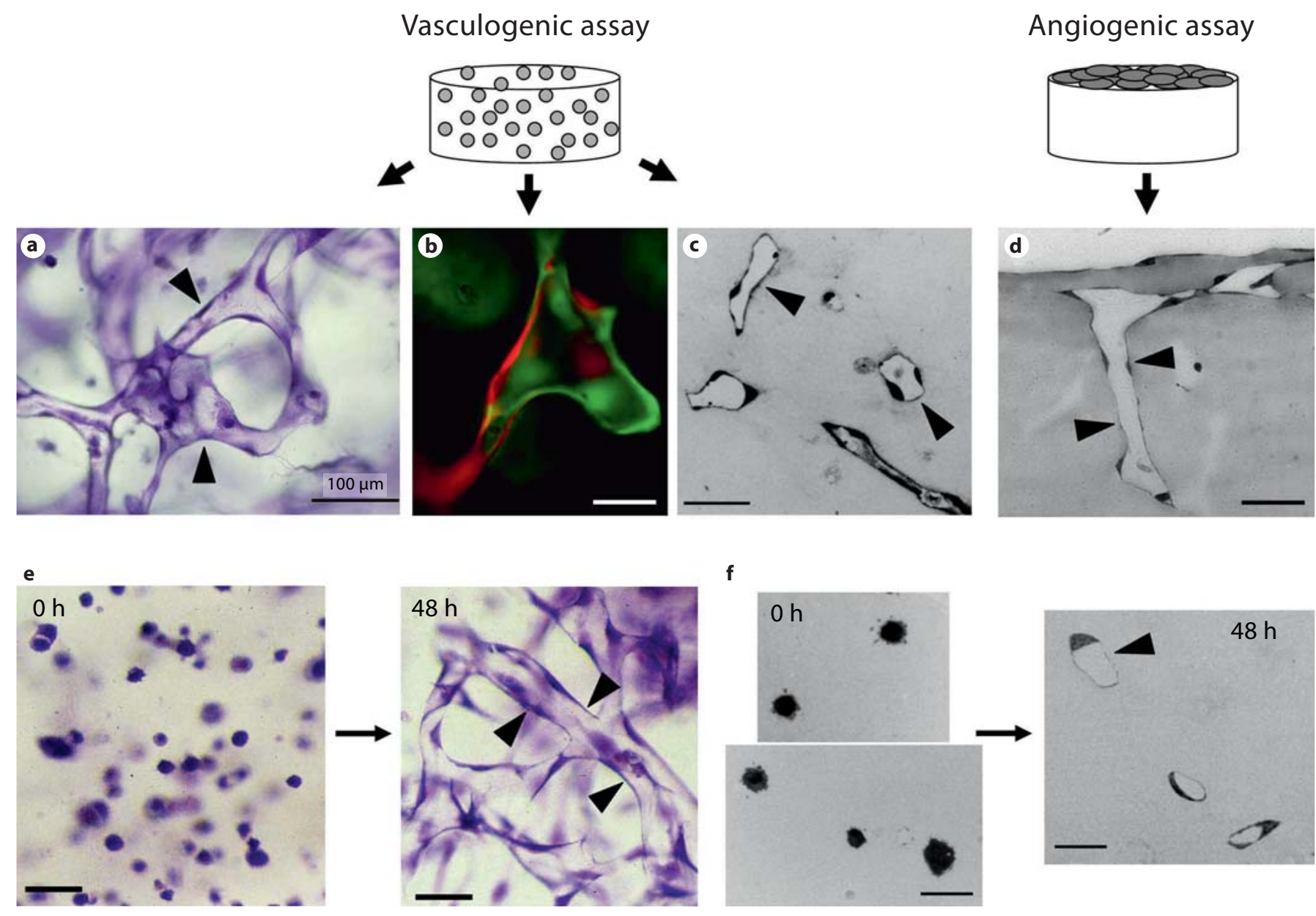

\section{Vasculogenic assay}

Fig. 1. EC tube morphogenesis during vasculogenesis and angiogenesis in 3D collagen matrices. Human ECs were seeded within or on $3 \mathrm{D}$ collagen matrices and were allowed to undergo tube morphogenesis and sprouting over time using assays that mimic the vasculogenesis or angiogenic sprouting. a-c, e, f Human ECs seeded within $3 \mathrm{D}$ collagen matrices and after $2(\mathbf{e}, \mathbf{f}), 3(\mathbf{b})$, or 5 (a, c) days were fixed and stained with toluidine blue $(\mathbf{a}, \mathbf{e})$ or were cross sectioned $(\mathbf{c}, \mathbf{f})$ and then photographed. b Human ECs car-

which distinguishes them from the basal membranes in contact with ECM.

Recently, controversy has arisen over whether this process even occurs in ECs [Blum et al., 2008; Strilic et al., 2009]. We have recently discussed these issues in detail in a separate review [Davis et al., 2011b] but, to summarize our previous discussion, it is clear that intracellular vacuolation is an important component of EC lumenogenesis in many vascular beds. These dynamic vacuole structures have been observed by multiple inves- rying GFP or mRFP were seeded together in equal numbers and allowed to form tubes for $72 \mathrm{~h}$; they were then photographed under fluorescence microscopy. $\mathbf{d}$ ECs were seeded on the surface of collagen matrices and were allowed to sprout for $48 \mathrm{~h}$; they were then cross sectioned to visualize tube structures. Arrowheads indicate lumen and tube structures. Scale bars $=100 \mu \mathrm{m}(\mathbf{a}), 25 \mu \mathrm{m}$ (b, c, f), and $50 \mu \mathrm{m}(\mathbf{d}, \mathbf{e})$. tigators and using different systems both in vitro and in vivo (fig. 3) [Davis and Camarillo, 1996; Yang et al., 1999; Bayless and Davis, 2002; Davis et al., 2002; Davis and Bayless, 2003; Egginton and Gerritsen, 2003; Stratman et al., 2009a; Liu et al., 2010; Wang et al., 2010; Zovein et al., 2010]. Furthermore, we recently reported the presence of EC intracellular vacuoles in quail ECs during tube assembly in the chorioallantoic membrane at embryonic day 5 [Stratman et al., 2009a] and we also show another example in figure 3e. During human EC tube formation 

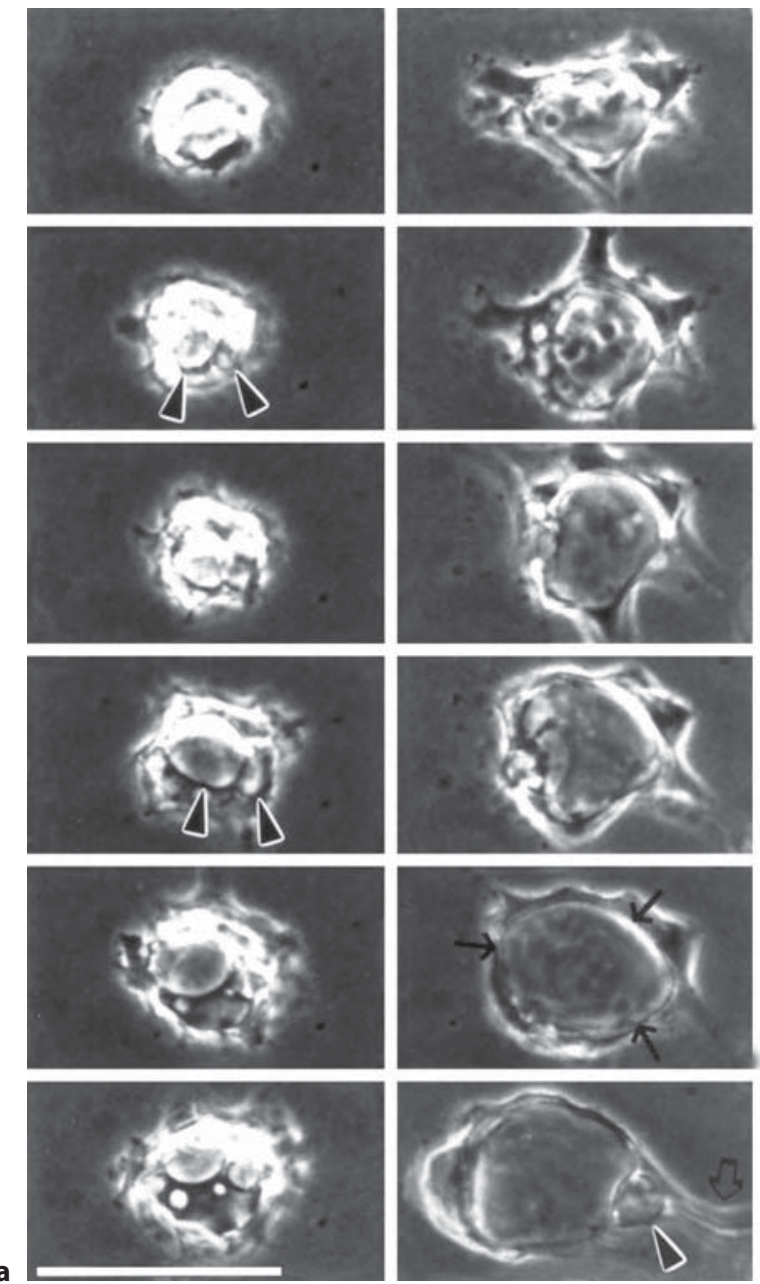
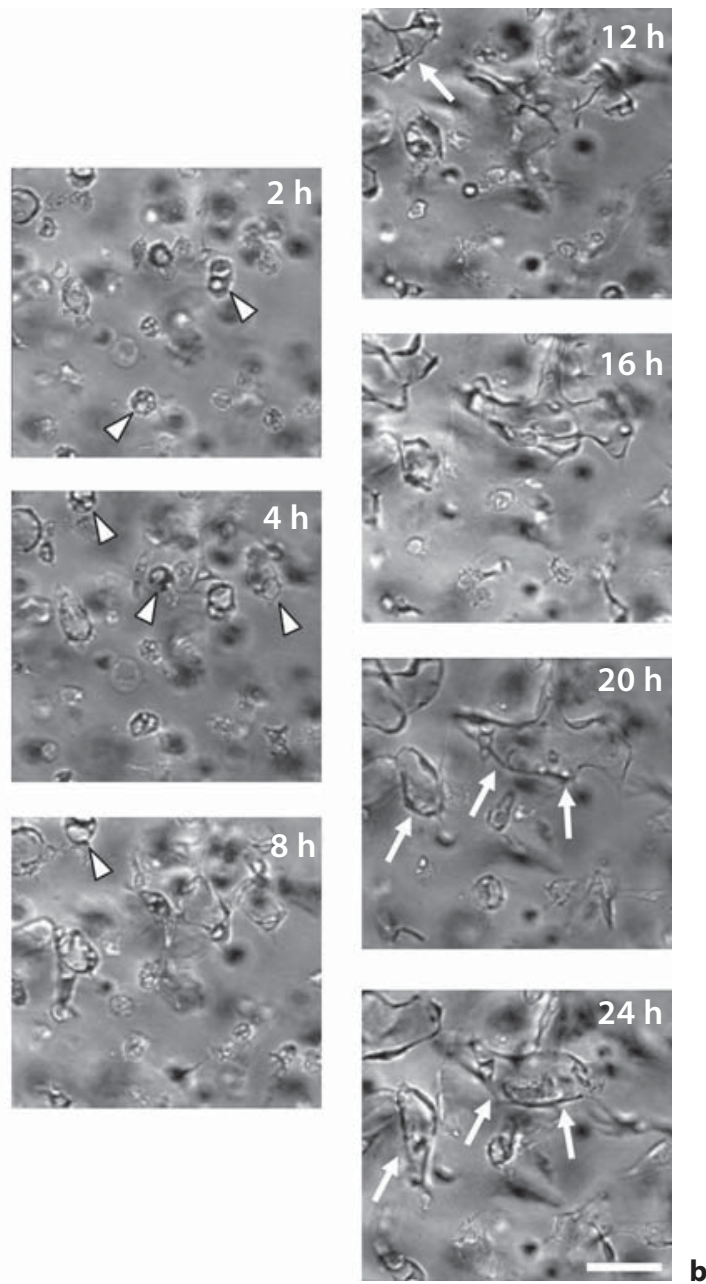

Fig. 2. Single ECs can form lumenal structures through intracellular vacuole formation and fusion as well as MT1-MMP-dependent lumen expansion in 3D collagen matrices. Real-time video analysis of EC lumen and tube formation was performed examining a single EC (a) versus multiple ECs (b) in 3D collagen matrices over a 24-hour period. Intracellular vacuoles are dynamically formed and through fusion events rapidly create apical membranes necessary for the accelerated events which underlie the EC lumen formation process. Arrowheads indicate intracellular vacuoles, arrows indicate the border of lumen structures, and the open arrow indicates an extended process to facilitate multicellular tube assembly. Scale bars $=25 \mu \mathrm{m}$ (a) and $50 \mu \mathrm{m}$ (b).

in vitro, it is a major mechanism controlling lumen formation and it is directly linked to integrin- and Cdc42/ Racl-mediating signaling which are major regulators of EC tubulogenesis in 3D matrices [Bayless and Davis, 2002; Koh et al., 2008a, 2009; Sacharidou et al., 2010]. Furthermore, GFP-tagged Cdc42 or Rac1 target to vacuole membranes and these targeting vectors have been very helpful to visualize them in vitro and in vivo (fig. 3) [Bayless and Davis, 2002; Kamei et al., 2006; Davis et al., 2007]. Additionally, GFP-RalA (fig. 3b) [Davis et al., 2007] and GFP-moesin (expressed in zebrafish) [Wang et al., 2010] have been shown to target to intracellular vacuole membranes during tube formation events. The GFP-Cdc42 fusion protein was expressed using an ECspecific promoter in zebrafish and it was shown that intracellular vacuoles were dynamically formed and participated in EC lumen formation during intersegmental sprouting events in vivo during vascular development [Kamei et al., 2006]. They could even be filled through extracellular application of fluorescent microspheres or dyes, a finding reproduced by two independent groups using zebrafish [Kamei et al., 2006; Wang et al., 2010]. 

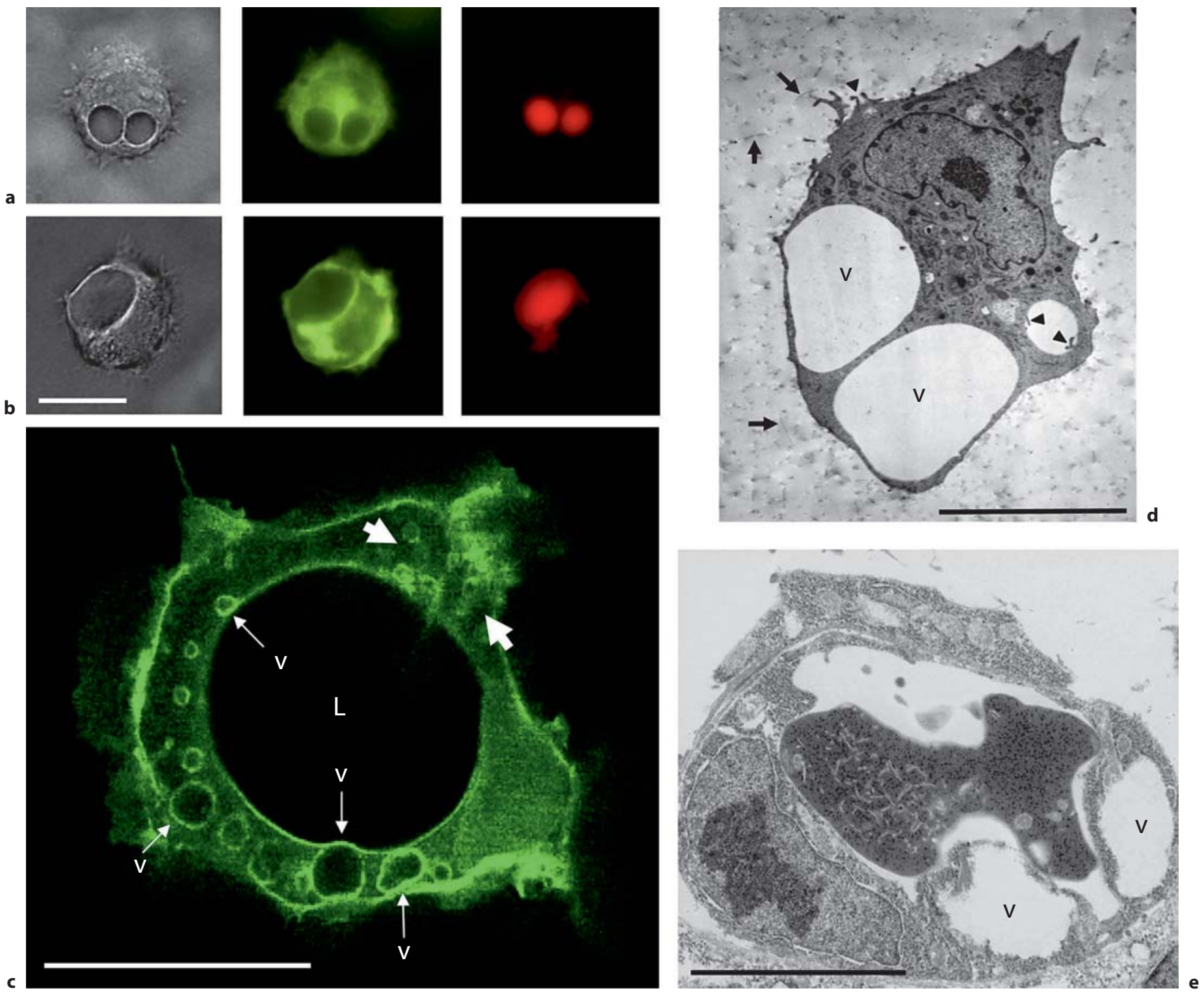

Fig. 3. EC intracellular vacuoles control lumen formation through rapid creation of apical membranes during EC tubulogenesis in vitro and in vivo in 3D matrices. a ECs were transfected with adenoviral vectors carrying GFP-Cdc42 (a), GFP-RalA (b), or GFPRacl (c) and ECs undergoing morphogenesis were photographed under fluorescence microscopy. In $\mathbf{a}$ and $\mathbf{b}$, carboxyrhodamine was added as an extracellular tracer to label structures that arise through pinocytic events such as the pinocytic intracellular vacuoles shown. Note the strong vacuole membrane labeling by GFPRalA (b) with lesser but detectable labeling with GFP-Cdc42 (a). c Strong intracellular labeling is noted with GFP-Racl in this 24- hour image where vacuoles appear to arise in the cell periphery (white arrows) and are transported toward the apical surface to fuse. The apical membrane (facing the lumenal compartment; L) of the developing tube is also labeled with GFP-Racl. v = Intracellular vacuoles. d, e Electron micrographs of ECs with intracellular vacuoles are shown from in vitro cultures (d) and developing EC tubes in vivo during quail vascular development (e). Arrows indicate collagen type I matrix and arrowheads indicate fine processes extending from the pinocytosed preapical vacuole membrane or EC cell surface. Scale bars $=15 \mu \mathrm{m}$ (a, b), $25 \mu \mathrm{m}$ (c), 10 $\mu \mathrm{m}(\mathbf{d})$, and $5 \mu \mathrm{m}(\mathbf{e})$. 


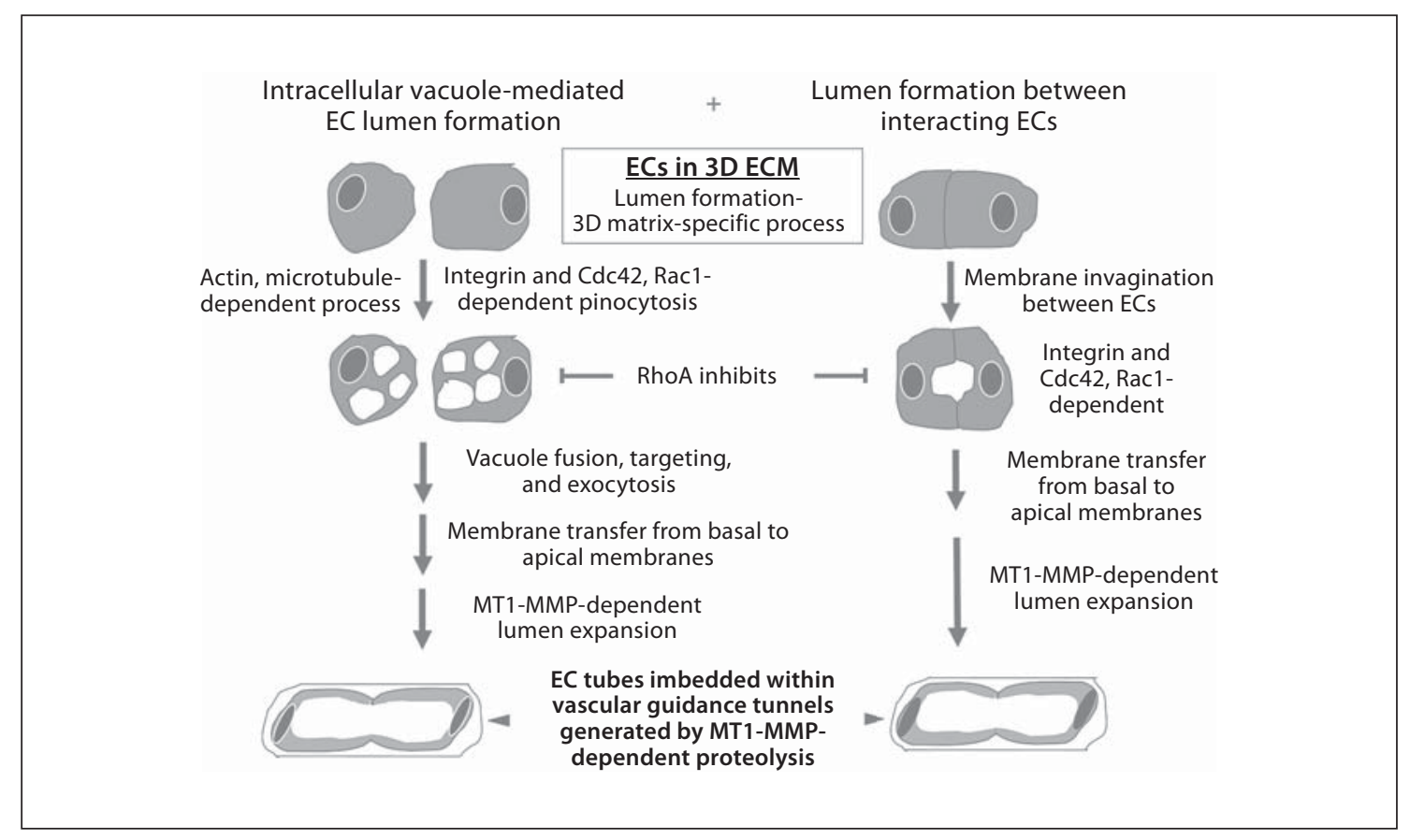

Fig. 4. Molecular mechanisms controlling EC lumen and tube assembly in 3D ECM environments. This schematic diagram illustrates two major mechanisms that control EC lumen and tube assembly during vascular morphogenesis. Importantly, the molecular and signaling requirements for these processes control a 3D matrix-specific event since lumen and tube formation do not occur on $2 \mathrm{D}$ surfaces. There are many common features in the two mechanisms including dependence on actin and microtubule function, integrins, dependence of Cdc42 and Racl, and inhibition of these events by RhoA. Furthermore, membrane invagination events oc- cur during both processes, with one revealing pinocytic structures (intracellular vacuoles) (left side) and the other invaginating membrane, but pinosomes are less prominent (right side). However, marked membrane transfer from basal membranes to apical membranes is key to both mechanisms as well as MT1-MMP-dependent lumen expansion which is required to create vascular guidance tunnels during the morphogenic process. Importantly, lumen formation mechanisms from ECs seeded as single cells versus preaggregated cells in 3D collagen matrices appear to be very similar in terms of both molecular and signaling requirements.
Thus, intracellular vacuoles appear to be similar to macropinosomes which were demonstrated in our past in vitro studies [Davis and Camarillo, 1996; Bayless and Davis, 2002; Davis et al., 2007] and which also demonstrated that the vacuoles undergo both intracellular fusion and exocytic fusion events with plasma membranes (both apical and basal) (fig. 3a, b).

Several studies have suggested or shown the absence of large intracellular vacuoles (few small vacuoles were observed in the mouse aorta) within the developing aorta despite the clear ability of these ECs to form lumen structures [Strilic et al., 2009; Wang et al., 2010]. Our previous studies in vitro have demonstrated that intracellular vacuoles are one, but not the only, mechanism utilized by ECs to form lumenal structures (fig. 4) [Davis et al., 2002, 2007, 2011b; Saunders et al., 2006; Stratman et al., 2009b; Sacharidou et al., 2010]. For example, we have shown that MT1-MMP appears to function primarily in
EC lumen expansion through required proteolytic events (and which create physical spaces in the ECM termed vascular guidance tunnels) (fig. 5) [Saunders et al., 2006; Stratman et al., 2009b; Sacharidou et al., 2010]. This same process occurs whether ECs are suspended as single cells or EC-EC aggregates (fewer vacuoles are observed in aggregates but they are not absent). Also, the activity of MT1-MMP appears to facilitate vacuole-vacuole fusion events to promote lumenal expansion, but blockade of MT1-MMP does allow for some intracellular vacuole formation events [Saunders et al., 2006; Stratman et al., 2009b; Sacharidou et al., 2010]. However, when MT1MMP is inhibited, lumens and tubes fail to form because the lumenal spaces require concomitant vascular guidance tunnel space formation (an MT1-MMP-dependent event) [Stratman et al., 2009b].

Additional work reveals that EC lumen signaling complexes [Sacharidou et al., 2010] as well as a previously de- 


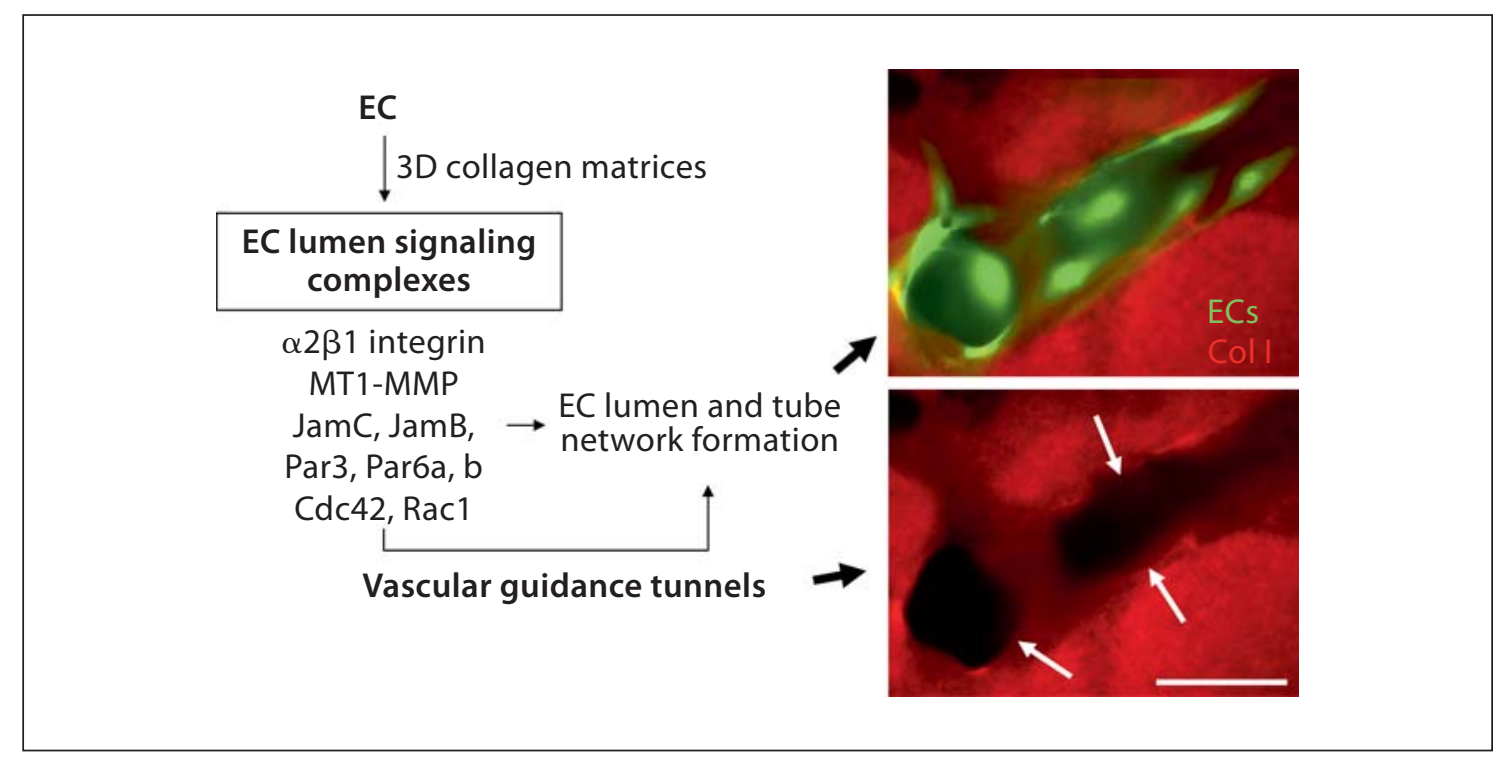

Fig. 5. EC lumen signaling complexes are critical regulators of EC tubulogenesis in 3D matrices by controlling both tube and vascular guidance tunnel formation. An EC lumen signaling complex consisting of the $\alpha 2 \beta 1$ integrin, MT1-MMP, JamC, JamB, Par3, Par6a and Par6b, Cdc42-GTP, and Rac1-GTP has been identified which is necessary for human ECs to form lumens and tube networks in 3D collagen matrices. GFP-labeled ECs were seeded in 3D collagen matrices, and after $24 \mathrm{~h}$ cultures were fixed and stained with anti-collagen type I antibodies and immunofluores-

scribed kinase cascade [Koh et al., 2008a, 2009] control EC lumen formation whether ECs are suspended as single cells or as preaggregated cells within $3 \mathrm{D}$ collagen matrices [Sacharidou et al., in preparation]. We preaggregated ECs in order to mimic conditions that would occur in large vessel assembly such as in the aorta. Importantly, Cdc42, and Racl, but not RhoA or Rho kinase [Bayless and Davis, 2002, 2004; Koh et al., 2008a; Sacharidou et al., 2010; Davis et al., 2011b], control EC lumen formation from single or aggregated ECs in 3D collagen matrices along with a2ß1, MT1-MMP, JamB, JamC, Par3, and Par6b [Sacharidou et al., in preparation]. There are a few subtle distinctions between single versus aggregated ECs in controlling EC lumen in that Pak-2 and Yes appear to be less critical when they are aggregated compared to single ECs but, in contrast, other kinases, protein kinase $\mathrm{C}(\mathrm{PKC}) \varepsilon$, Src, and atypical PKC (aPKC) (i.e. PKCiota) are required during these processes [Sacharidou et al., in preparation]. Importantly, the major findings demonstrate that the critical EC lumen signaling complex and its downstream effectors [Sacharidou et al., 2010] are necessary whether ECs are single or aggregated cells and thus strongly suggests that cent images were prepared. Arrows indicate the borders of vascular guidance tunnels that are created as a result of the EC lumen signaling complex which contains the collagenolytic MMP, MT1MMP. Vascular guidance tunnels represent matrix conduits that are created as a result of the EC tube formation process and which play a key role in vessel assembly, remodeling, and EC-mural cell interactions that are important during later steps of vascular tube maturation. Scale bar $=25 \mu \mathrm{m}$.

the molecular and signaling requirements for lumen and tube assembly are likely to be very similar in different vascular beds of varying diameter and location. Clearly, this is an important area for future investigation.

\section{Fundamental Role for ECM and Integrins in EC Lumen Formation and Tube Stabilization}

EC interactions with 3D ECM are essential in order for these cells to migrate, invade, and assemble into tube networks (fig. 1) [Davis and Senger, 2005; Davis et al., 2007, 2011b; Hynes, 2007, 2009; Rhodes and Simons, 2007; Somanath et al., 2009; Arroyo and Iruela-Arispe, 2010; Warren and Iruela-Arispe, 2010; Senger and Davis, 2011]. Importantly, EC tubulogenesis is a $3 \mathrm{D}$ matrix-specific event which does not occur on 2D ECM surfaces (fig. 4). During vasculogenesis, individual ECs locate each other within 3D matrices requiring process extension, cell shape changes including intracellular vacuole formation coupled with lumen formation, EC surface-directed proteolysis to direct motility and lumen expansion and then mul- 
ticellular tube assembly (fig. 1, 2, 4). In all cases, integrin contacts with the ECM are required for these events in 3D matrices. Two types of ECM appear to be particularly conducive to EC tube morphogenesis and sprouting and they include collagen type I and fibrin matrices [Davis and Senger, 2005; Senger and Davis, 2011]. Interestingly, the $\alpha 2 \beta 1$ integrin controls EC tube formation and sprouting in collagen matrices [Davis and Camarillo, 1996; Senger et al., 1997; Bayless and Davis, 2003] while $\alpha 5 \beta 1$ and $\alpha v \beta 3$ control these events in fibrin matrices [Bayless et al., 2000; Bayless and Davis, 2003; Carnevale et al., 2007]; thus, the integrins involved in vascular morphogenesis are directly linked to the ECM environment in which the process is occurring. These integrins have also been shown to be involved in regulating EC tube morphogenesis during developmental and adult neovascularization events [Drake et al., 1992; Senger et al., 1997; Stupack and Cheresh, 2004; San Antonio et al., 2009; van der Flier et al., 2010; Zovein et al., 2010]. In contrast, basement membrane matrices containing laminin variants (i.e. laminins $511,521,411$, and 421), collagen type IV, and tissue inhibitor of metalloproteinase (TIMP)-3 appear to provide inhibitory signals to EC tube morphogenesis but, interestingly, contribute to the development of vascular tube maturation, stabilization, and EC quiescence [Liu and Senger, 2004; Davis and Senger, 2005; Saunders et al., 2006; Stratman et al., 2009a, 2010; Senger and Davis, 2011].

More recent work has shown an exciting new aspect of integrin function during these events and this has been revealed comparing EC-only tubulogenesis to EC tubulogenesis in the presence of pericytes in $3 \mathrm{D}$ collagen matrices. We recently reported that EC-pericyte heterotypic cell interactions are necessary for vascular basement membrane matrix assembly in vitro and in vivo during quail vascular development [Stratman et al., 2009a, 2010]. In the absence of pericytes, EC-only cultures fail to assemble a vascular basement membrane matrix. Importantly, blocking antibodies directed to the integrins $\alpha 5 \beta 1, \alpha 3 \beta 1, \alpha 6 \beta 1$, and $\alpha 1 \beta 1$ (fibronectin, laminin, nidogen and collagen type IV receptors) only have inhibitory effects when ECs and pericytes are cocultured [Stratman et al., 2009a]. In contrast, $\alpha 2 \beta 1$ blocking antibodies markedly interfere with EC tube formation as well as stability in EC-only cultures [Stratman et al., 2009a]. Interestingly, in EC-pericyte cocultures, the influence of $\alpha 2 \beta 1$ diminished with time as the collagen type I matrix was remodeled with vascular basement membrane matrix underlying EC tubes [Stratman et al., 2009a]. $\alpha 5 \beta 1, \alpha 3 \beta 1, \alpha 6 \beta 1$, and $\alpha 1 \beta 1$ all interact with basement membrane components; this ap- pears to be the reason why they now become functionally relevant selectively in EC-pericyte cocultures, which is coincident with the deposition of vascular basement membrane matrix (representing the ECM ligands for these integrins). Previous studies focusing on vascular development have strongly implicated $\alpha 5 \beta 1$ and fibronectin matrix deposition during these events [Francis et al., 2002; Astrof et al., 2007; Hynes, 2007; Astrof and Hynes, 2009; van der Flier et al., 2010]. EC-only tubes become much wider with time in the absence of basement membrane deposition compared to EC-pericyte tubes with basement membrane [Stratman et al., 2009a, 2010]. This increased width depends on MMP activity, and inhibition of MT1-MMP appears to be one mechanism whereby EC-pericyte interactions lead to decreased tube width [Saunders et al., 2006; Stratman et al., 2009a, b]. Inhibitors of tube morphogenesis appear to be present in vascular basement membranes such as laminins and TIMP-3 [Bayless and Davis, 2003; Liu and Senger, 2004; Saunders et al., 2006]. Interestingly, we have preliminary data that laminin addition to 3D collagen matrices blocks EC lumen and tube formation and, furthermore, that laminin receptors such as $\alpha 6 \beta 1$ appear to be involved in this inhibitory response [Sacharidou and Davis, unpubl. observations]. TIMP-3, which is produced by pericytes and which is an ECM-binding TIMP, blocks EC tube formation through inhibition of MT1-MMP [Saunders et al., 2006]. Thus, the ECM remodeling process during EC-pericyte tube coassembly limits vascular morphogenesis and leads to tube stabilization in part through inhibition of tube formation mechanisms. A second mechanism by which pericytes affect tube stabilization is by preventing proregressive stimuli (e.g. MMP-1 and MMP-10) [Saunders et al., 2005; Chang et al., 2006] from collapsing and regressing EC tube networks. This latter mechanism appears to be controlled by EC-derived TIMP-2 and pericyte-derived TIMP-3 [Davis and Saunders, 2006; Saunders et al., 2006; Stratman et al., 2009a].

\section{Fundamental Role for the Rho GTPases Cdc42 and Rac1 in EC Lumen and Tube Formation; in Contrast, RhoA Controls Vascular Tube Collapse and Regression}

Integrin binding to ECM leads to Rho GTPase activation and this activation mechanism is a fundamental step in the ability of ECs to form lumens and tubes in 3D ECM (fig. 4). This is the case whether isolated ECs undergo in- 
Table 1. Key regulators of vascular lumen formation in 3D matrices (I)

\begin{tabular}{|c|c|c|c|}
\hline $\mathrm{Cdc} 42$ & $\begin{array}{l}\text { Regulator for actin and microtubule } \\
\text { cytoskeletons to control lumen formation; } \\
\text { binds to effectors such as Pak- } 2 \text { and Pak- } 4 \\
\text { and Par6 isoforms; its activation depends on } \\
\text { EC lumen signaling complexes in 3D } \\
\text { matrices }\end{array}$ & $\begin{array}{l}\text { Many effectors including Pak-2, Pak-4, } \\
\text { and Par6b which play a role in lumen } \\
\text { formation; present in EC lumen signaling } \\
\text { complexes with integrins, MT1-MMP, } \\
\text { JamB, JamC, Par3, and Par6b }\end{array}$ & $\begin{array}{l}\text { Bayless and Davis, 2002; } \\
\text { Koh et al., 2008a, 2009; } \\
\text { Sacharidou et al., 2010; } \\
\text { Hoang et al., 2010b }\end{array}$ \\
\hline Integrins & $\begin{array}{l}\text { Regulate actin and microtubule } \\
\text { cytoskeletons to control lumen formation; } \\
\text { particular integrins involved relate to the } \\
\text { ECM environment where tube formation } \\
\text { occurs; influence EC Par3 expression to } \\
\text { control lumenogenesis }\end{array}$ & $\begin{array}{l}\text { Key component of EC lumen signaling } \\
\text { complexes; } \alpha 2 \beta 1 \text { and MT1-MMP interact } \\
\text { to coordinate EC-collagen interactions } \\
\text { and MT1-MMP-dependent proteolysis } \\
\text { that controls EC lumen formation }\end{array}$ & $\begin{array}{l}\text { Drake et al., 1992; } \\
\text { Davis and Camarillo, 1996; } \\
\text { Senger et al., 1997; } \\
\text { Bayless et al., 2000; } \\
\text { Sacharidou et al., 2010; } \\
\text { Zovein et al., 2010 }\end{array}$ \\
\hline MT1-MMP & $\begin{array}{l}\text { Regulates EC surface proteolysis in } \\
\text { collagen and fibrin matrices to control lumen } \\
\text { formation; involved in the generation of } \\
\text { vascular guidance tunnels which regulate EC } \\
\text { tube remodeling and EC tube maturation } \\
\text { events in 3D matrices }\end{array}$ & $\begin{array}{l}\text { EC lumen signaling complexes containing } \\
\text { MT1-MMP control EC lumen formation; } \\
\text { also coordinates Cdc42 activation and } \\
\text { downstream kinase signaling cascades } \\
\text { necessary for lumen formation }\end{array}$ & $\begin{array}{l}\text { Lafleur et al., 2002; } \\
\text { Chun et al., 2004; } \\
\text { Saunders et al; 2006; } \\
\text { Stratman et al., 2009b; } \\
\text { Sacharidou et al., } 2010\end{array}$ \\
\hline JamB/JamC & $\begin{array}{l}\text { JamB and JamC form heterodimeric } \\
\text { complexes that regulate EC lumen } \\
\text { formation; interact with the lumen regulator } \\
\text { Par3; present in EC lumen signaling } \\
\text { complexes that control Cdc } 42 \text { activation in } \\
\text { 3D but not 2D matrices }\end{array}$ & $\begin{array}{l}\text { The JamB and JamC cytoplasmic tails are } \\
\text { necessary for Par3 binding, Cdc } 42 \\
\text { activation, and EC lumen formation in 3D } \\
\text { matrices; JamB and JamC are components } \\
\text { of EC lumen signaling complexes }\end{array}$ & Sacharidou et al., 2010 \\
\hline
\end{tabular}

tracellular vacuole formation to form lumens or ECs are interacting with each other (fig. 4). Although, EC adhesion to 2D ECM surfaces leads to Rho GTPase activation, this does not lead to lumen formation, which is a $3 \mathrm{D} \mathrm{ma-}$ trix-specific process [Davis et al., 2002, 2007, 2011b; Sacharidou et al., 2010]. This is an important issue that needs to be considered in terms of why EC lumen formation and tubulogenesis occurs in a 3D matrix-specific manner and not on 2D surfaces. Our laboratory first described that Cdc42 controls EC lumen formation (table 1) [Bayless and Davis, 2002]. A dominant negative inhibitor of Cdc42 completely blocked lumen formation of human ECs in 3D collagen matrices [Bayless and Davis, 2002]. Later, we demonstrated that siRNA suppression of Cdc 42 also completely abrogates lumen and tube formation [Davis et al., 2007; Koh et al., 2008a]. Dominant negative Racl and siRNA directed to Racl also markedly blocked lumen formation, while blockade of RhoA had no inhibitory influence [Bayless and Davis, 2002; Davis et al., 2007; Koh et al., 2008a]. Thus, EC lumen formation in 3D matrices depends on Cdc42 and Racl, but not RhoA. In support of these findings, both Cdc42 and Racl have been implicated 
in vivo during either vascular developmental or postnatal vascularization responses [Tan et al., 2008; Hoang et al., $2010 \mathrm{a}, \mathrm{b}]$. Of great interest is the observation that RhoA controlled vascular tube collapse following microtubule depolymerization [Bayless and Davis, 2004]. Thus, RhoA was actually an inhibitor of the process and actively participated in EC tube collapse (fig. 4) [Bayless and Davis, 2004]. We initially reported that expression of constitutively active RhoA in ECs completely blocked their ability to form intracellular vacuoles and lumens (fig. 4) [Bayless and Davis, 2002]. Later work by several groups demonstrated an important role for a downstream effector of RhoA, Rho kinase (ROCK), in mediating tube collapse and regression [Mavria et al., 2006; Im and Kazlauskas, 2007]. Interestingly, they reported that Src and Erk1/2 activation led to inhibition of ROCK. We recently demonstrated that Cdc42- and Racl-mediated signaling during EC lumen formation induces marked activation of both Src and Erk1/2 [Koh et al., 2009; Sacharidou et al., 2010], suggesting that this pathway would not only induce the formation of tube networks but at the same time prevent tube collapse by blocking RhoA and ROCK activation. Blockade of Src or Erk1/2 completely interferes with EC lumen formation, thus indicating that $\mathrm{Cdc} 42 / \mathrm{Racl}$ actively opposes the action of RhoA and vice versa [Koh et al., 2009; Sacharidou et al., 2010].

Interestingly, siRNA suppression of cerebral cavernous malformation (CCM) 1 or CCM2 leads to blockade of EC lumen formation (table 2) [Whitehead et al., 2009; Lampugnani et al., 2010] but is also accompanied by marked activation of RhoA and ROCK [Glading et al., 2007; Kleaveland et al., 2009; Whitehead et al., 2009; Stockton et al., 2010] which leads to disassembly of ECEC junctional contacts. Recently, we completed an interesting experiment where siRNA suppression of either CCM1 or CCM2 was performed at the same time as siRNA suppression of RhoA. These results demonstrate that suppression of RhoA expression completely rescues the inhibitory influence of CCM1 or CCM2 knockdown on EC lumen and tube formation [Sacharidou et al., in preparation]. Furthermore, we have confirmed this result by expressing dominant negative RhoA in CCM1 or CCM2 siRNA-treated ECs which also completely rescues the lumen formation defect in these treated cells [Sacharidou et al., in preparation]. Thus, much of the influence of CCM1 and CCM2 on EC lumen formation appears to be related to their ability to inhibit RhoA activation during the lumen formation process. The details of how these events occur at a molecular level are actively being pursued by our group as well as others.
An important function of Rho GTPases is to activate downstream effectors that then regulate their biological influence. Using an siRNA screening strategy, we identified p21-activated kinase (Pak)-2 and Pak- 4 as well as the polarity protein Par6b as key effectors that controlled EC lumen formation [Koh et al., 2008a; Sacharidou et al., 2010; Davis et al., 2011b]. We further demonstrated that dominant negative mutants of both Pak-2 and Pak-4 completely inhibited EC lumen formation in 3D collagen matrices. Importantly, we showed that Pak-2 and Pak-4 activation (as indicated by phosphorylation) directly correlated with the time course of EC lumen formation [Koh et al., 2008a]. In addition, we demonstrated that Cdc42GTP could be shown to be interacting with both activated Pak-2 and Pak-4 in ECs undergoing tube formation in 3D collagen matrices [Koh et al., 2008a]. Also, we demonstrated that both Par6b and its binding partner, Par3, could be shown to be interacting with Cdc42-GTP containing complexes during the tube formation process [Koh et al., 2008a; Sacharidou et al., 2010]. Previous work implicated Pak-1 in these events [Kiosses et al., 2002]. Furthermore, a study implicated a functional role for Pak-2 in zebrafish vascular development [Liu et al., 2007] while another study demonstrated a role for Pak-4 during mouse vascular development [Galan Moya et al., 2009; Tian et al., 2009]. Interestingly, Pak-4 has been reported to inhibit the activity of several Rho guanine exchange factors (GEFs) [Callow et al., 2005] and thus can decrease RhoA activation (an inhibitor of EC lumen formation) (fig. 4) [Bayless and Davis, 2002, 2004; Mavria et al., 2006; Im and Kazlauskas, 2007].

Cdc42-GTP is part of an EC lumen signaling complex that contains Par6b, Par3, junction adhesion molecule (Jam)B, JamC, MT1-MMP, and the $\alpha 2 \beta 1$ integrin (fig. 5) [Sacharidou et al., 2010]. Recent studies indicate that Par6a and Racl are also part of this complex [Sacharidou et al., in preparation]. Together, this complex of proteins controls human EC lumen formation and directly regulates Cdc42 activation in 3D collagen matrices but not on 2D collagen surfaces [Sacharidou et al., 2010]. In addition, EC motility was selectively inhibited in 3D matrices but not on 2D matrix surfaces when the expression of each of the EC lumen signaling complex components was blocked by siRNA suppression. A key regulator of these events is MT1-MMP, which selectively controls 3D matrix motility due to its proteolytic activity (since ECM is a barrier to cell motility in 3D matrices but not 2D matrices) (fig. 5) [Stratman et al., 2009b]. siRNA suppression of MT1-MMP or blockade of its activity with chemical or protein inhibitors selectively blocked EC motility and lumen formation in 
Table 2. Key regulators of vascular lumen formation in $3 \mathrm{D}$ matrices (II)

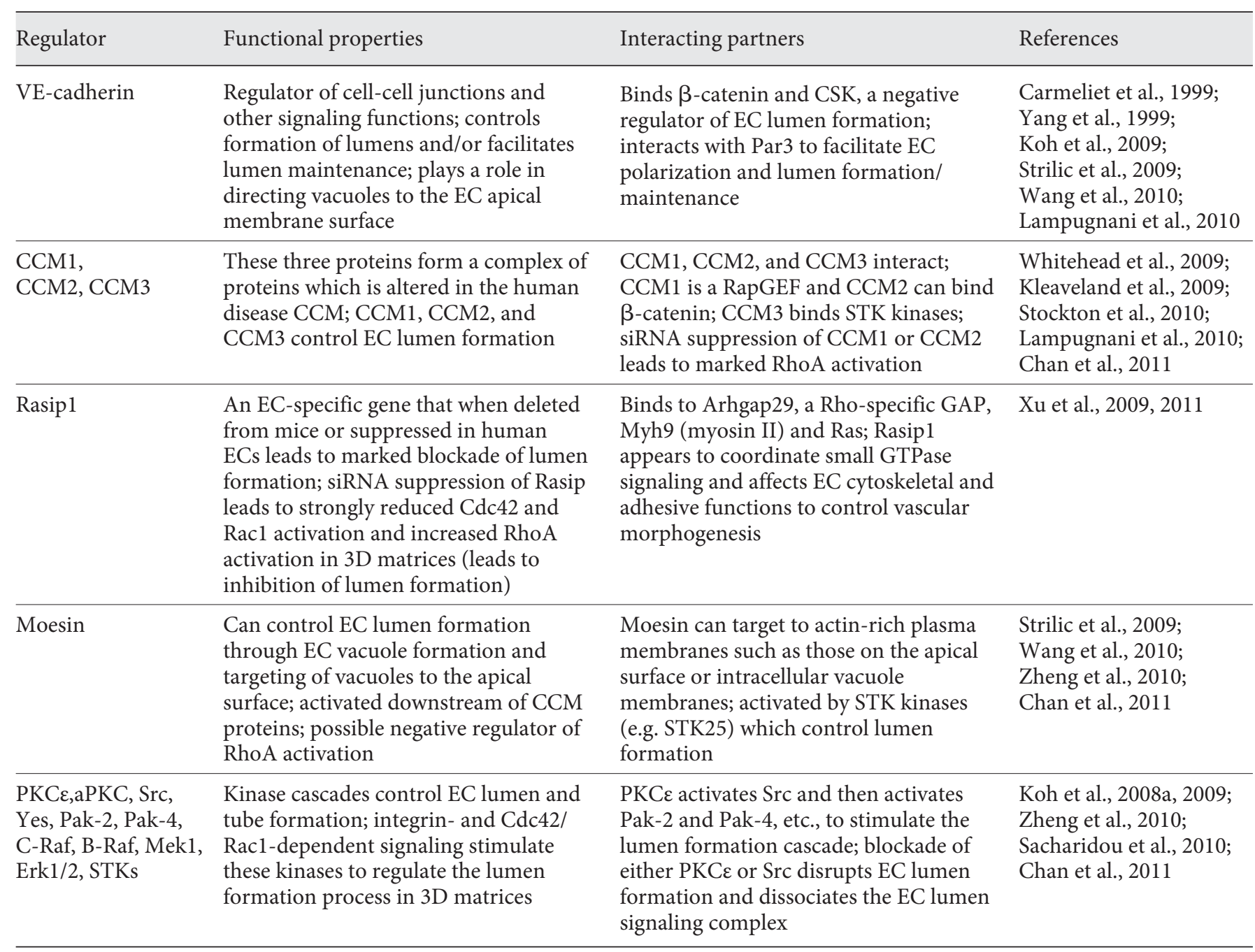

3D collagen matrices (fig. 6) [Stratman et al., 2009b]. EC motility in $3 \mathrm{D}$ matrices is necessary for EC lumen and multicellular tube assembly (through generation of vascular guidance tunnels), and thus MT1-MMP proteolytic activity is required for these events [Saunders et al., 2006; Stratman et al., 2009b]. Importantly, MT1-MMP activity also plays a critical role in controlling Cdc42 activation in $3 \mathrm{D}$ collagen matrices but not on $2 \mathrm{D}$ collagen surfaces [Sacharidou et al., 2010]. Thus, the EC lumen signaling complex is required for each of the key steps in EC lumen and tube formation including: (i) EC interactions with ECM through integrins, (ii) ECM proteolysis to create vascular guidance tunnels and promote cell motility, (iii) activation of Cdc42 and Racl and suppression of RhoA to control cytoskeletal rearrangements and Par3-dependent polarity signaling, (iv) membrane trafficking events to transfer membrane components from basal to apical surfaces (i.e. intracellular vacuoles and other vesicle trafficking), and (v) activation of protein kinase cascades including PKC $\varepsilon$, aPKC, Src, Yes, C-Raf, B-Raf, and Erk1/2 which together play critical roles in controlling these events (fig. 4, 5) [Davis et al., 2007, 2011b; Sacharidou et al., 2010]. In this latter case, considerable effort will be required to identify the particular kinase targets as well as target residues that are phosphorylated by these kinases to affect the EC lumen and tube formation process.

There is considerable evidence that the lumen regulators identified using in vitro model systems are also operative during these events in vivo (tables 1,2). Key regulators that have been identified using knockout mice, morpholino oligos in zebrafish, or zebrafish mutants include Rac1, Pak-2, Pak-4, $\alpha 2 \beta 1$ integrin, $\beta 1$ integrins, 

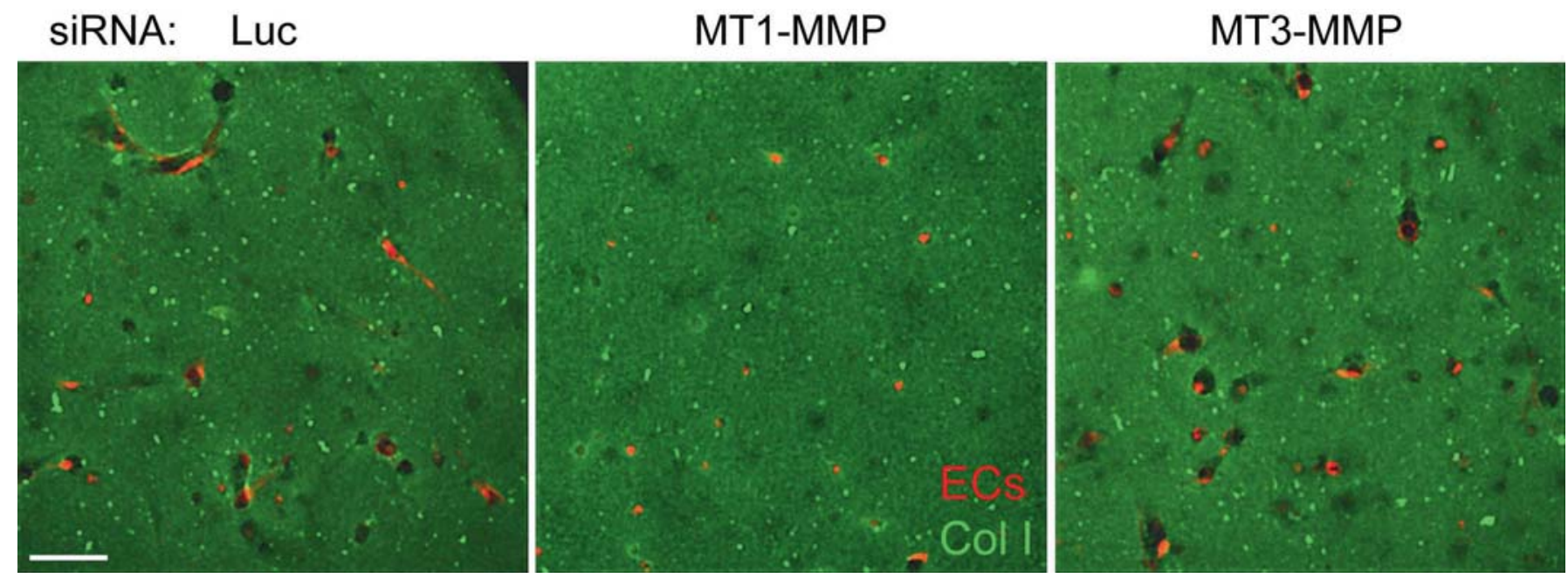

Fig. 6. MT1-MMP is required for EC lumen and tube formation in 3D collagen matrices. siRNA suppression experiments were performed using mRFP-labeled human ECs and three siRNAs which were control luciferase (Luc), MT1-MMP, and MT3-MMP. The treated ECs were seeded in FITC-labeled 3D collagen matrices and, after $24 \mathrm{~h}$, cultures were imaged by confocal fluorescence microscopy. Scale bar $=100 \mu \mathrm{m}$.

Par3, CCM1, CCM2, and CCM3 (tables 1, 2). A novel regulator of EC lumen formation that has just been reported is Rasipl, an EC-specific gene that shows EC specificity in mice and Xenopus species [Xu et al., 2009, 2011]. Knockout of Rasip1 leads to complete failure of EC lumen formation in all vascular beds from the aorta to the microvasculature [Xu et al., 2011]. Importantly, Rasip1 was found to associate with Arhgap29, a GTPase activating protein with specificity for inactivating RhoA-GTP. Interestingly, knockout of either Rasip1 or Arhgap29 leads to marked RhoA activation of ECs in either 2D or 3D matrices. SiRNA suppression of either Rasip1 or ArhGAP29 markedly blocks EC lumen and tube formation in 3D collagen matrices and leads to suppression of Cdc42 and Racl activation, while there is a strong increase in RhoA activation [Xu et al., 2011]. Furthermore, knockdown of Rasipl blocks the kinase cascade controlling EC lumen formation including suppression of Src, Pak-4, C-Raf, B-Raf, and Erk1/2 [Xu et al., 2011]. Interestingly, when Rasip1 was knocked down, it was possible to rescue this lumen formation defect when dominant negative RhoA or siRNA to RhoA were introduced into human ECs in 3D collagen matrices [Xu et al., 2011]. Thus, a major function of Rasip1 is to coordinate Rho GTPase signaling and thereby affect EC morphogenesis, cytoskeletal organization, and integrin-mediated adhesion. It also facilitates Cdc42 and Racl activation while it suppresses RhoA activation to control the lumen and tube formation process in $3 \mathrm{D}$ matrices.

\section{Cell Polarity and Membrane Trafficking Mechanisms in the Molecular Control of EC Lumen and Tube Formation and Stabilization}

As discussed above, membrane trafficking plays a key role in intracellular vacuole formation and fusion mechanisms that affect lumen formation (fig. 4). Also, it is a critical process for trafficking of membrane from the basal surface to the apical surface during lumen formation. Thus, in order for lumen formation to occur, there needs to be a marked transfer of membrane components (i.e. lipids, proteins, etc.) to the apical membrane to expand this surface. Interestingly, DNA microarray analysis has revealed marked increases in cholesterol biosynthetic enzymes (e.g. HMG CoA reductase) during EC lumen formation [Bell et al., 2001], which is consistent with increased membrane requirements such as the creation of the apical membrane surface.

Basal-to-apical transfer of membrane has been demonstrated during the process of epithelial cell lumen formation where apical and basal membrane polarity is much more defined with respect to the polarized distribution of apical transmembrane proteins (e.g. podocalyxin and annexin2) as well as membrane-associated cytoskeletal components (e.g. actin and moesin) compared to EC lumen formation [Bryant and Mostov, 2008; Andrew and Ewald, 2010; Bryant et al., 2010; Datta et al., 2011]. Recent work reveals a role for Cdc42-dependent 
polarity, Par-3, annexin2, PTEN, and Rabs 11A and 8A in epithelial membrane polarity during lumen and tube formation events [Martin-Belmonte et al., 2007, 2008; Jaffe et al., 2008; Bryant et al., 2010; Hao et al., 2010; Datta et al., 2011]. The latter two Rab proteins and their associated proteins play a key role in membrane trafficking as well as apical and basolateral sorting of protein and membrane cargo during epithelial tube morphogenesis and function [Bryant et al., 2010]. An important point is that many epithelial cell tubes are specialized for apical secretion or absorption, and thus there is a critical dependence on sorting of specific transmembrane proteins and cytoskeletal components to accomplish this specific sorting process [Bryant and Mostov, 2008]. EC-lined tubes are primarily conduits for blood flow and gas exchange and have less secretory or absorptive functions; thus, their need for such specialized polarity as observed in epithelial tubes appears to be much less.

We would like to suggest that EC polarity may be accomplished in different ways that are uniquely related to the functional differences of EC-lined tubes compared to other types of tubes. For example, one clear distinction of EC and epithelial tubes is that mural cells are recruited to EC tubes in most cases and that they recruit selectively to the abluminal EC surface (fig. 7, 8). Furthermore, mural cells such as pericytes regulate the deposition of vascular basement membrane matrix selectively on this abluminal surface, again demonstrating a polarized process (fig. 8, 9) [Stratman et al., 2009a, 2010]. Furthermore, flow forces selectively affect the EC apical surface (a fluid interface) and together with the basal contact with vascular basement membrane (a matrix interface) likely play major roles in EC polarity signaling and control (fig. 8). The relative lack of EC apicalbasal transmembrane protein or cytoskeletal markers may make the case that polarization of such markers might not be that relevant to the lumen formation process. The rapidity with which EC form lumens and tubes, without much evidence for apical or basal transmembrane protein polarity markers, suggests that this property may not be a central feature underlying the mechanistic requirements for lumen formation. Perhaps ECs and epithelial cells are distinct in this regard. Another possibility is that polarized molecules in ECs will be discovered as more information is obtained regarding this process. However, it is of great interest that known polarity regulators such as Cdc42, Par6b, Par3, and $\mathrm{aPKC}$ are fundamental regulators of EC lumen formation; thus, polarity signaling pathways are necessary

Vascular Lumen Formation in 3D Matrices for EC lumen formation [Koh et al., 2008a; Sacharidou et al., 2010; Zovein et al., 2010; Davis et al., 2011b]. Also, these components are required molecules within EC lumen signaling complexes that are necessary for the EC lumen and tube formation process (fig. 5) [Sacharidou et al., 2010].

\section{JamB and JamC Interact with the Polarity Protein Par3 to Control the Assembly of EC Lumen Signaling Complexes Which Are Required for Tubulogenesis in 3D Matrices}

Critical components of the EC lumen signaling complex are JamB and JamC [Sacharidou et al., 2010] (fig. 5), which were previously implicated in tight junction formation and signaling as well as inflammation [Ebnet et al., 2003, 2004]. JamC has been implicated in angiogenic responses although its specific functional purpose during these events was unclear [Lamagna et al., 2005]. A recent study demonstrated mutations in JamC that lead to neonatal lethality in humans due to vascular abnormalities and hemorrhage, particularly in the central nervous system [Mochida et al., 2011]. JamB and JamC are known to interact with each other and, through their cytoplasmic tails, are known to bind the polarity protein Par3 [Ebnet et al., 2003, 2004]. siRNA suppression of both JamB and JamC markedly blocks EC lumen formation as well as the addition of soluble blocking antibodies to both JamB and JamC [Sacharidou et al., 2010]. Combined blockade of both molecules (using siRNAs or blocking antibodies) results in dramatic inhibition of EC lumen formation [Sacharidou et al., 2010]. This inhibition is accompanied by a lack of Cdc42 activation, blockade of MT1-MMP-mediated tunnel formation, and complete blockade of the kinase cascade (i.e. reduced Src, Pak-2, Pak-4, B-Raf, C-Raf, and Erk1/2 phosphorylation) that is activated downstream of the EC lumen signaling complex and which is necessary for EC tubulogenesis in 3D matrices [Sacharidou et al., 2010].

Importantly, increased expression of either JamB or JamC constructs without their cytoplasmic tails leads to marked blockade of tube formation [Sacharidou et al., 2010]. Deletion of the C-terminal 5 amino acids of the JamC cytoplasmic tail which is known to bind Par3 also completely inhibits EC lumen formation in $3 \mathrm{D}$ collagen matrices. The expression of these constructs also blocked Cdc 42 activation in $3 \mathrm{D}$ collagen matrices but, importantly, not on 2D collagen substrates; this is an indication that 

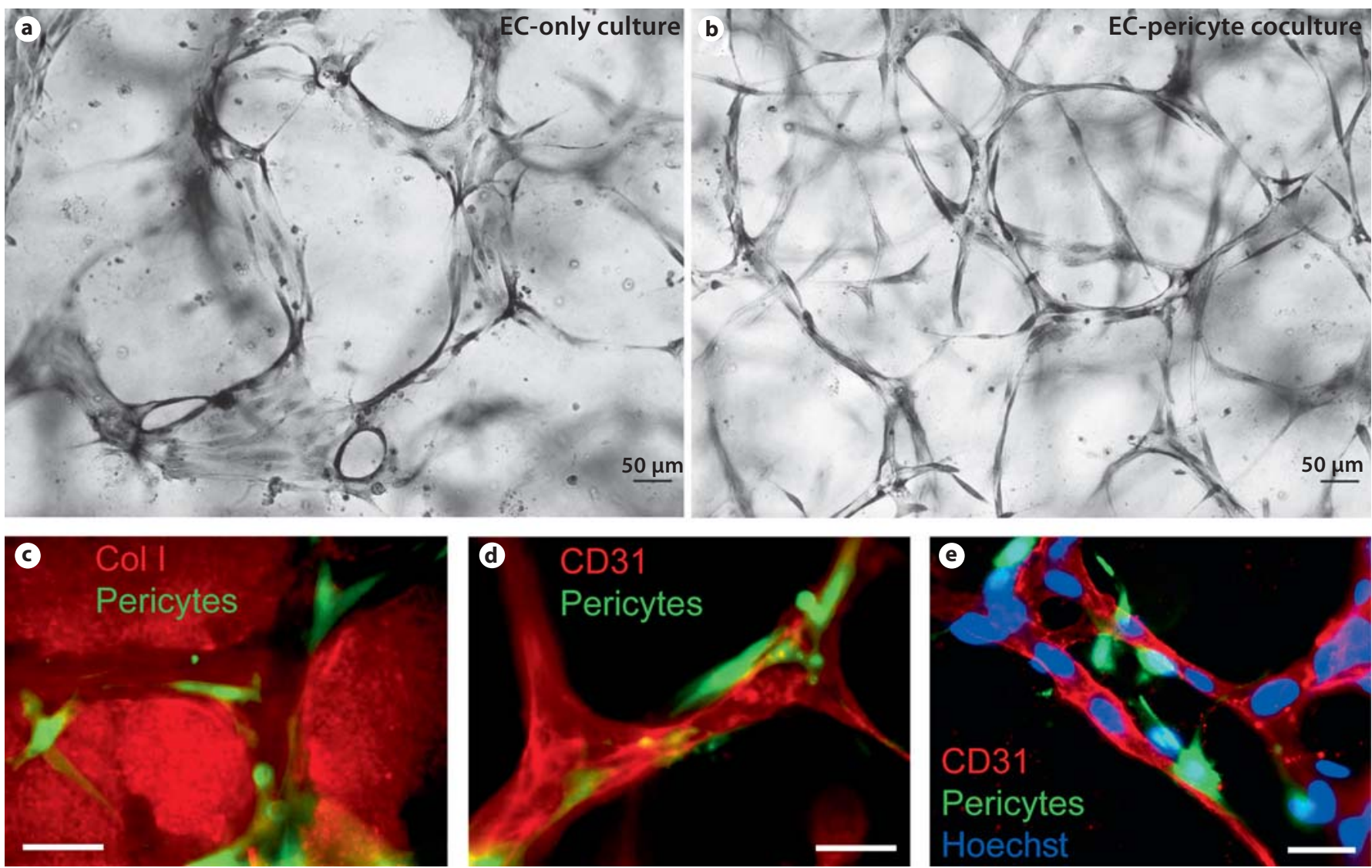

Fig. 7. Recruitment of pericytes to EC-lined tubes occurs within vascular guidance tunnels that are generated during the EC tubulogenic process in 3D ECM. EC-only (a) or EC-pericyte (b) cultures were established in 3D collagen matrices, and after 5 days cultures were fixed, stained, and photographed. Note that the presence of pericytes creates much more uniformity and complex patterns in the EC tube networks than in their absence and EC tube widths are markedly increased in EC-only versus EC-pericyte cocultures. c-e ECs were seeded with GFP-pericytes and allowed to assemble for 5 days. After fixation, cultures were stained with collagen type I antibodies to identify vascular guidance tunnels (c), CD31 to identify EC tubes (d), and CD31 to label ECs, but also with the addition of Hoechst dye to label both EC and pericyte nuclei. Scale bars $=50 \mu \mathrm{m}(\mathbf{a}, \mathbf{b})$ and $25 \mu \mathrm{m}(\mathbf{c}-\mathbf{e})$.
$\mathrm{JamB} / \mathrm{JamC}$ interactions with Par3 are necessary for EC Cdc42 activation in 3D matrices [Sacharidou et al., 2010]. In addition, we demonstrated that increased expression of JamA interfered with lumen formation while siRNA suppression of JamA increased lumen formation, which suggests that JamA inhibits this process [Sacharidou et al., 2010]. The mechanism by which this occurs is not clear; however, it may be through competition with Par3 binding which could disrupt the EC lumen signaling complex and prevent tube formation. Thus, JamB and JamC appear to play a central role in EC lumenogenesis by controlling the assembly and function of EC lumen signaling complexes [Sacharidou et al., 2010; Davis et al., 2011b].

\section{Critical Functional Role for MT1-MMP-Mediated Proteolysis and Vascular Guidance Tunnel Formation during EC Tube Assembly and Maturation in 3D ECM}

Considerable information demonstrates that cell surface proteolysis is a major functional regulator of EC tubulogenesis and invasive phenomena such as sprouting [Lafleur et al., 2002; Chun et al., 2004; Saunders et al., 2006; Sabeh et al., 2009; Stratman et al., 2009b]. MT1MMP is a key transmembrane proteinase that mediates many of these cellular behaviors [Fisher et al., 2009; Sabeh et al., 2009]. A consequence of these proteolytic events during tubulogenesis or invasion is the creation of physical spaces within the ECM termed vascular guid- 
Fig. 8. EC-pericyte interactions control vascular basement membrane matrix assembly. Electron micrographs are shown illustrating how EC-pericyte contact and intercellular communication control vascular basement membrane assembly in 3D collagen matrices. EC-pericyte cocultures (a-c) or EC-only cultures (c) were fixed and processed for transmission electron microscopy after 5 days of culture. a Ongoing basement membrane assembly (arrowheads) underlying ECs is shown and also a pericyte likely migrating on the EC abluminal surface within vascular guidance tunnels to stimulate continuous basement membrane deposition along EC tubes. b Continuous basement membrane underlying an EC tube with an associated pericyte. The collagen type I matrix is indicated. c Ongoing vascular basement membrane matrix formation that is stimulated by EC-pericyte interactions during tube coassembly events. d EC-only culture which shows a lumenal surface but no underlying basement membrane matrix when pericytes are not present. The collagen type I matrix is indicated. Scale bar = $0.5 \mu \mathrm{m}(\mathbf{a}, \mathbf{c}, \mathbf{d})$ and $2 \mu \mathrm{m}(\mathbf{b})$.

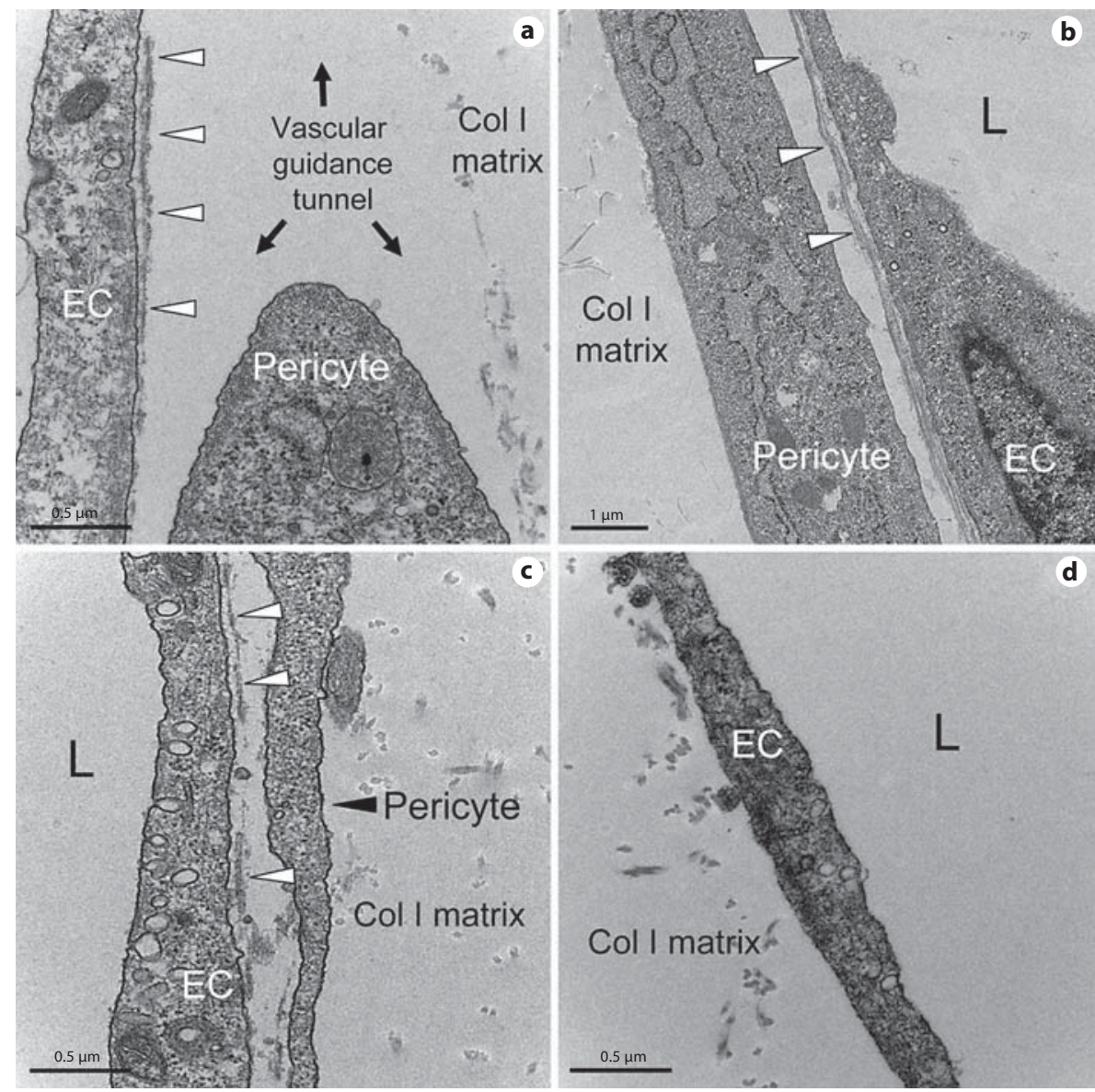

ance tunnels (fig. 5). Both blood and lymphatic ECs demonstrate this morphogenic requirement in $3 \mathrm{D}$ collagen matrices (fig. 6, 10). Single cells such as tumor cells, pericytes, and fibroblasts also create tunnel spaces that trail the invading cells [Fisher et al., 2009; Sabeh et al., 2009]. Importantly, we have observed that all regulators of tubulogenesis that we have identified also directly affect the generation of vascular guidance tunnels mediated through MT1-MMP proteolytic activity coordinated with integrin-, JamB/C/Par3/Par6b-, and Cdc42/Rac1mediated signaling (i.e. EC lumen signaling complexes) (fig. 6) [Sacharidou et al., 2010]. The coordination of tubulogenesis with cell surface proteolysis is one of the key reasons why tube formation is a $3 \mathrm{D}$-specific event, since such proteolysis is not required for EC motility on $2 \mathrm{D}$ matrix surfaces.

Initial experiments demonstrated that addition of TIMP-2, TIMP-3, and TIMP-4, but not TIMP-1, could block either EC lumen formation or EC sprouting events [Bayless and Davis, 2003; Saunders et al., 2006; Stratman et al., 2009b]. Also, the chemical inhibitor GM6001 blocked both processes [Saunders et al., 2006]. SiRNA suppression screening experiments revealed a major role for MT1-MMP, with a lesser but detectable role for MT2MMP in both EC lumen formation and EC sprouting into 3D collagen matrices (fig. 6) [Saunders et al., 2006; Stratman et al., 2009b]. Increased expression of wild-type but not catalytically inactive recombinant MT1-MMP leads to a marked increase in EC lumen formation as well as vascular guidance tunnel formation [Stratman et al., 2009b]. Interestingly, expression of a wild-type MT1MMP recombinant construct without its cytoplasmic tail leads to a marked increase in EC lumen and tunnel formation [Sacharidou et al., 2010]. Previous reports describe that the cytoplasmic tail of MT1-MMP mediates its internalization from the cell surface and thereby negatively regulates cell surface proteolytic activity. These findings are consistent with our results in that increased EC lumen and tunnel formation was accompanied by increased cell surface expression of MT1-MMP in the ab- 


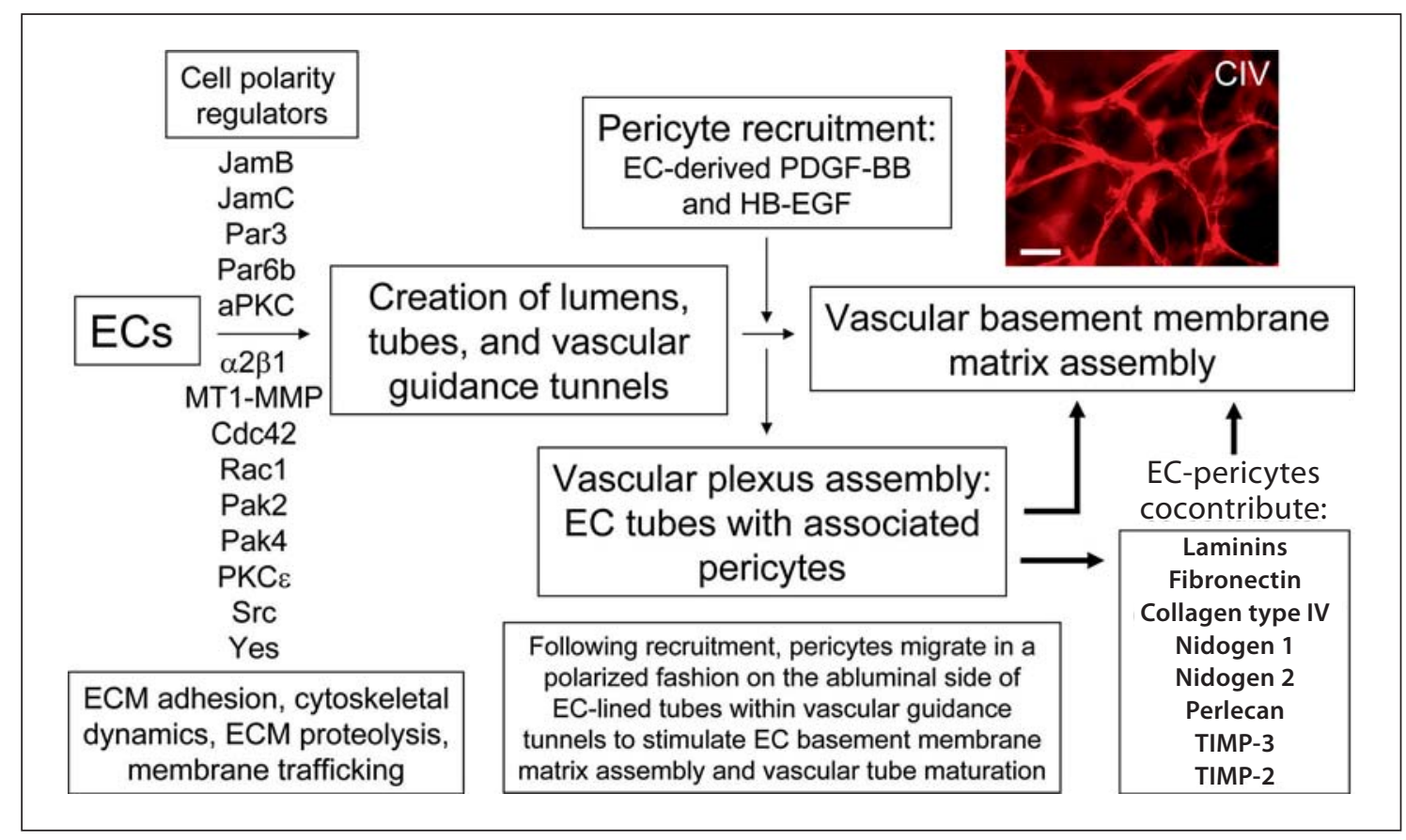

Fig. 9. Identification of molecule and signaling requirements for EC tubulogenesis and EC-pericyte tube coassembly which leads to vascular basement membrane matrix assembly and tube maturation. Key steps in vascular tube morphogenesis as well as pericyte recruitment and vascular basement membrane matrix assembly are illustrated. In addition, specific molecules are listed that are required for EC tube morphogenesis, as well as pericyte recruitment to tubes and basement membrane assembly events. The upper right panel illustrates collagen type IV (CIV) deposition as an indicator of basement membrane formation that occurs following EC and pericyte interactions and cocontribution of the indicated basement membrane matrix components. Scale bar $=50 \mu \mathrm{m}$.

sence of its cytoplasmic tail. Of great interest was our finding that expression of catalytically inactive MT1MMP without its cytoplasmic domain results in dominant negative inhibition of endogenous MT1-MMP with complete blockade of lumen and vascular guidance tunnel formation [Sacharidou et al., 2010]. Finally, MT1MMP, a membrane-tethered collagenase with specificity for triple-helical collagens, strongly interacts with the collagen-binding integrin $\alpha 2 \beta 1$, and together they are components of the EC lumen signaling complex which is required for $\mathrm{EC}$ tubulogenesis in $3 \mathrm{D}$ collagen matrices [Sacharidou et al., 2010].

An important consequence of EC tubulogenesis is the generation of vascular guidance tunnels, which are critical matrix conduits that control vascular tube remodeling, cell motility, and ECM remodeling events that are necessary for tube maturation and stabilization (fig. 9). In fact, pericytes which are recruited along the abluminal surface of EC-lined tubes are actually recruited into vascular guidance tunnels in which the tubes are embedded (fig. 4, 7). In this way, pericytes are able to migrate on this abluminal surface and, in conjunction with ECs, induce the deposition of a continuous vascular basement membrane (fig. 8, 9) [Stratman et al., 2009a]. Interestingly, once vascular guidance tunnels are created, ECs are able to freely migrate through these matrix conduits in an MMP-independent manner [Stratman et al., 2009b]. Also, once pericytes are recruited through EC-derived PDGF-BB and HB-EGF to the EC tubes within the tunnel spaces [Stratman et al., 2009a, 2010], they are also able to migrate on the EC abluminal surface in an MMP-independent manner to facilitate the vascular basement membrane matrix assembly process [Stratman et al., 2009a]. Thus, vascular guidance tunnels, which are downstream of MT1-MMP-dependent proteolysis, play a major role in a fundamental step in vascular tube remodeling and maturation (i.e. vascular basement membrane assembly secondary to dynamic EC-pericyte interactions) that is required for blood vessel structure and function during development and postnatal life (fig. 5, 9). 
Fig. 10. Lymphangiogenic sprouting and lumen formation requires MMP activity in 3D collagen matrices. Human dermal lymphatic ECs were seeded on the surface of collagen gels and angiogenic sprouting was stimulated by sphingosine-1-phosphate which was incorporated into the collagen matrix. Culture media contained reduced serum supplement, phorbol ester, FGF-2, and VEGF-C to facilitate survival and morphogenesis. In addition, GM6001 was added at $5 \mu \mathrm{M}$ to block MMP activity (b) or was not added (a). Arrows indicate lymphatic lumen and tube structures. Lymphatic ECs were shown to stain with Prox-1 and LYVE-1 (c, d) and were also shown to attach to anti-podoplanin antibodies coated on plastic wells, while human umbilical vein ECs failed to attach under the same conditions. Overall, these results confirmed their identity as lymphatic ECs. Scale bars $=100 \mu \mathrm{m}(\mathbf{a}, \mathbf{b})$ and $25 \mu \mathrm{m}(\mathbf{c}, \mathbf{d})$.
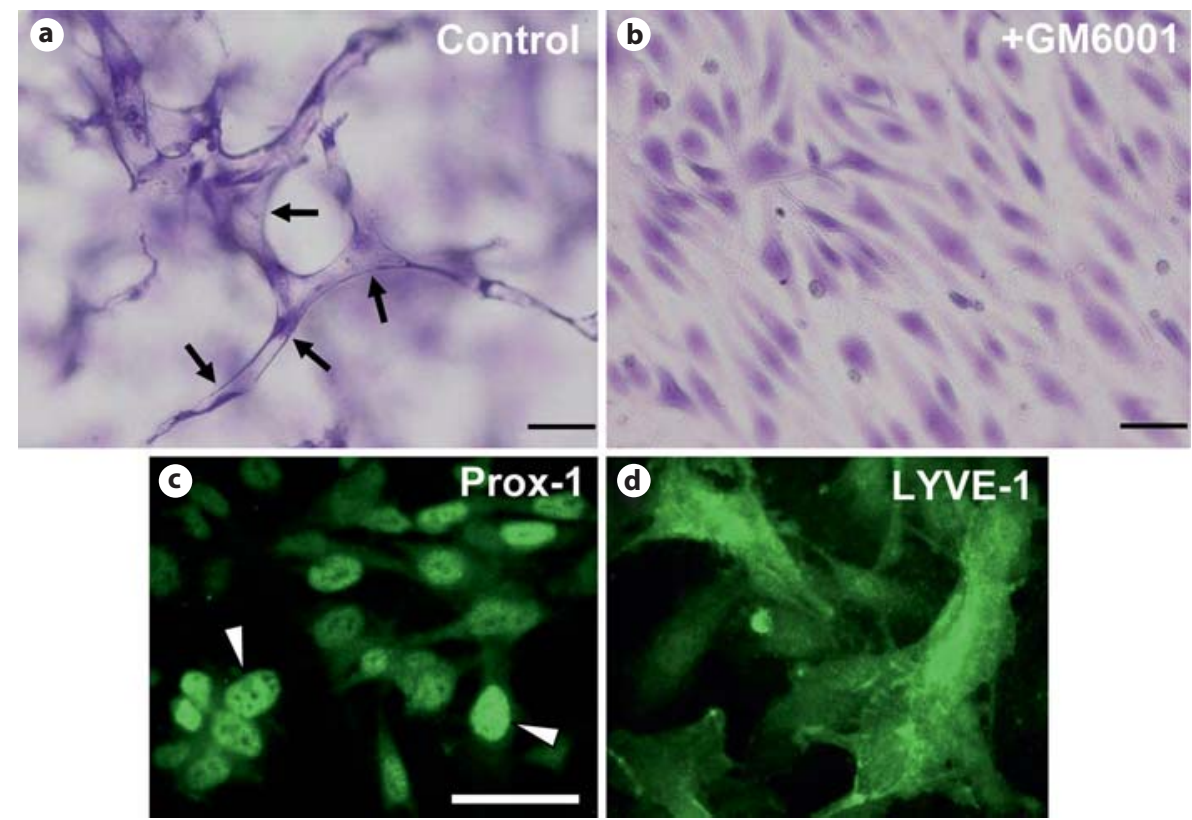

\section{Endothelial Lumen Signaling Complexes Control Vascular Lumen Formation and Tube Morphogenesis Specifically in 3D Matrix Environments}

Considerable past work has revealed the critical role of individual molecules such as integrins, Rho GTPases, polarity molecules such as Par3, and MT1-MMP, but there was previously little understanding of how these molecules work together to control these events (tables 1,2) [Davis et al., 2007, 2011b]. The discovery of EC lumen signaling complexes, which represent multiprotein complexes containing Cdc42-GTP, Par6b, Par3, JamB, JamC, MT1-MMP, and $\alpha 2 \beta 1$ leads to a better mechanistic understanding of how molecules work together to control a complex process such as EC tubulogenesis in a 3D matrix environment [Sacharidou et al., 2010; Davis et al., 2011b]. There appears to be a critical interdependence to control the 3D matrix-specific tube formation process [Sacharidou et al., 2010; Davis et al., 2011b]. As an example, we demonstrated that MT1-MMP activity is necessary for Cdc42 activation in 3D collagen matrices and vice versa in that blockade of $\mathrm{Cdc} 42$ leads to markedly reduced MT1-MMP activity (as indicated by a lack of lumen and vascular guidance tunnel formation) [Sacharidou et al., 2010]. In contrast, the molecules do not appear to influence each other when ECs are seeded on the surface of collagen matrices; thus, the Cdc42 and MT1-MMP inter- dependence requires that ECs be present in a 3D matrix environment [Sacharidou et al., 2010]. Also, RhoA activation was not affected by either molecule regardless of whether the cells were cultured in $3 \mathrm{D}$ or on $2 \mathrm{D}$ matrices. Also, we demonstrated that the JamB and JamC cytoplasmic tails were necessary for Cdc42 activation, MT1-MMP activity (to create tunnel spaces), and EC lumen formation in $3 \mathrm{D}$ matrices but not on $2 \mathrm{D}$ surfaces [Sacharidou et al., 2010]. Overall, these data indicate that the EC lumen signaling complex controls a 3D matrix-specific EC signaling pathway that is essential for ECs to form lumens and tubes during vascular morphogenic events. Furthermore, the EC lumen signaling complex is regulated by and controls a kinase signaling cascade involving $\mathrm{PKC} \varepsilon$, Src, Yes, Pak-2, Pak-4, C-Raf, B-Raf, Mek1, and Erk1/2 [Sacharidou et al., 2010]. Interestingly, PKC $\varepsilon$ or Src blockade leads to the disassembly of EC lumen signaling complexes [Sacharidou et al., unpubl. observations], which suggests that these kinases play a major role in maintaining the interactions necessary to promote EC lumen formation in 3D matrices. aPKC isoforms such as PKCiota [Sacharidou et al., in preparation] play a role in EC lumen formation through direct interactions with Par6 isoforms. Finally, kinases downstream of CCM1, CCM2, and CCM3 activation termed sterile 20 kinases (STKs) (e.g. STK25) work together to control EC lumenogenesis [Zheng et al., 2010; Chan et al., 2011]. Moesin, a regulator of lumen formation [Wang et al., 2010], appears to be a 
kinase substrate of particular STKs [Zheng et al., 2010]. A major future direction will be to pursue phosphoproteomic strategies to identify phosphorylated targets downstream of the key lumen regulating kinases that control these events.

\section{Conclusions and Future Directions}

It is clear that considerable progress has been made regarding the molecular basis for EC lumen and tube formation during vasculogenic and angiogenic events. This progress has been strongly enhanced by the development of molecular and signaling approaches to studying these events in vitro under defined conditions in $3 \mathrm{D}$ matrices. There are now numerous examples which have shown that particular in vitro tubulogenic systems have very accurately identified critical molecules that control vascular morphogenesis and maturation and which have been confirmed using mouse, avian, or zebrafish in vivo models. Increasingly, collaborative interactions between groups that specialize in in vivo versus in vitro morphogenic systems have strongly advanced our understanding of these processes.

Future studies should place a strong emphasis on how protein kinase cascades control vascular morphogenesis and how they interface with the EC lumen signaling complex as well as the microtubule and actin cytoskeletons to affect these events [Davis et al., 2011b; Senger and Davis, 2011]. Also, considerable work needs to be performed on how (and which) cytokines control particular steps in vascular morphogenesis and how ECM affect these responses [Davis and Senger, 2008; Hynes, 2009; Chen et al., 2010; Senger and Davis, 2011; Stratman et al., 2011]. Since cytokines and growth factors have specific affinities for particular ECM components [Hynes, 2009; Senger and Davis, 2011], cosignaling events between growth factor/cytokine receptors and ECM receptors such as integrins [Hynes, 2009; Somanath et al., 2009; Senger and Davis, 2011] will likely create unique signals that characterize the various steps of vascular tube morphogenesis, sprouting, and maturation.

Recent work using human ECs under serum-free defined conditions revealed some surprising results regarding the cytokines that control vascular tube morphogenesis in $3 \mathrm{D}$ matrices. We have reported that hematopoietic stem cell cytokines, stem cell factor (SCF), interleukin-3 (IL-3), and stromal-derived factor (SDF)$1 \alpha$, when combined, were able to stimulate vascular tube morphogenesis in 3D matrices [Stratman et al., 2011].
This occurred with or without pericyte addition, and pericytes were recruited to developing tubes to stimulate vascular basement membrane assembly in the presence of these defined hematopoietic cytokines [Stratman et al., 2009a, 2011]. In contrast, the combination of VEGF and FGF when mixed under these conditions was unable to support EC tube formation even in the presence of pericytes [Stratman et al., 2011]. Interestingly, this study also reported that VEGF and FGF can prime (or prepare) EC responsiveness to hematopoietic cytokines (through upregulation of hematopoietic cytokine receptors) [Stratman et al., 2011]. Thus, VEGF and FGF were particularly effective as EC priming factors, while the hematopoietic factors failed to show activity as priming factors but were strong promorphogenic factors. Thus, the optimal sequence of factor addition for maximal EC tube morphogenesis is to prime ECs with VEGF and FGF, followed by exposure to hematopoietic cytokines during the process of tube formation; reversing the order does not allow for EC tube morphogenesis [Stratman et al., 2011]. This study is one example of how in vitro models can be utilized to address complex biological questions in vascular biology. The work identified multiple novel regulators of vascular tube morphogenesis (i.e. hematopoietic stem cell cytokines stimulating EC tubulogenesis) and revealed new functional roles for previously described factors that influence vascularization responses (i.e. VEGF and FGF as EC priming factors preparing ECs for subsequent morphogenic signals) [Stratman et al., 2011]. These new insights could lead to novel therapeutic strategies that affect the vasculature in a positive or negative way in various disease states such as cancer, diabetes, and macular degeneration. It is also clear that such insights into the molecular requirements for EC tubulogenesis and maturation events can lead to new approaches for tissue bioengineering through the ability to generate human microvascular networks which could be integrated into specific tissue microenvironments (e.g. skin and heart) for transplantation and other applications.

\section{Acknowledgements}

This work was supported by NIH grants HL59373, HL79460, HL87308, and HL105606 to G.E.D. 


\section{References}

-Adams, R.H., K. Alitalo (2007) Molecular regulation of angiogenesis and lymphangiogenesis. Nat Rev Mol Cell Biol 8: 464-478.

Andrew, D.J., A.J. Ewald (2010) Morphogenesis of epithelial tubes: Insights into tube formation, elongation, and elaboration. Dev Biol 341: 34-55.

Aplin, A.C., E. Fogel, P. Zorzi, R.F. Nicosia (2008) The aortic ring model of angiogenesis. Methods Enzymol 443: 119-136.

-Armulik, A., A. Abramsson, C. Betsholtz (2005) Endothelial/pericyte interactions. Circ Res 97: 512-523.

-Arroyo, A.G., M.L. Iruela-Arispe (2010) Extracellular matrix, inflammation, and the angiogenic response. Cardiovasc Res 86: 226 235.

Astrof, S., D. Crowley, R.O. Hynes (2007) Multiple cardiovascular defects caused by the absence of alternatively spliced segments of fibronectin. Dev Biol 311: 11-24.

-Astrof, S., R.O. Hynes (2009) Fibronectins in vascular morphogenesis. Angiogenesis 12: 165-175.

Bayless, K.J., G.E. Davis (2002) The Cdc42 and Rac1 GTPases are required for capillary lumen formation in three-dimensional extracellular matrices. J Cell Sci 115: 1123-1136.

Bayless, K.J., G.E. Davis (2003) Sphingosine-1phosphate markedly induces matrix metalloproteinase and integrin-dependent human endothelial cell invasion and lumen formation in three-dimensional collagen and fibrin matrices. Biochem Biophys Res Commun 312: 903-913.

- Bayless, K.J., G.E. Davis (2004) Microtubule depolymerization rapidly collapses capillary tube networks in vitro and angiogenic vessels in vivo through the small GTPase Rho. J Biol Chem 279: 11686-11695.

Bayless, K.J., R. Salazar, G.E. Davis (2000) RGDdependent vacuolation and lumen formation observed during endothelial cell morphogenesis in three-dimensional fibrin matrices involves the alpha(v)beta(3) and alpha(5)beta(1) integrins. Am J Pathol 156: 1673-1683

Bell, S.E., A. Mavila, R. Salazar, K.J. Bayless, S. Kanagala, S.A. Maxwell, G.E. Davis (2001) Differential gene expression during capillary morphogenesis in $3 \mathrm{D}$ collagen matrices: regulated expression of genes involved in basement membrane matrix assembly, cell cycle progression, cellular differentiation and G-protein signaling. J Cell Sci 114: 2755 2773.

Blum, Y., H.G. Belting, E. Ellertsdottir, L. Herwig, F. Luders, M. Affolter (2008) Complex cell rearrangements during intersegmental vessel sprouting and vessel fusion in the zebrafish embryo. Dev Biol 316: 312-322.

Bryant, D.M., A. Datta, A.E. Rodriguez-Fraticelli, J. Peranen, F. Martin-Belmonte, K.E. Mostov (2010) A molecular network for de novo generation of the apical surface and lumen. Nat Cell Biol 12: 1035-1045.
Bryant, D.M., K.E. Mostov (2008) From cells to organs: building polarized tissue. Nat Rev Mol Cell Biol 9: 887-901.

Callow, M.G., S. Zozulya, M.L. Gishizky, B. Jallal, T. Smeal (2005) PAK4 mediates morphological changes through the regulation of GEF-H1. J Cell Sci 118: 1861-1872.

Carmeliet, P., M.G. Lampugnani, L. Moons, F. Breviario, V. Compernolle, F. Bono, G. Balconi, R. Spagnuolo, B. Oosthuyse, M. Dewerchin, A. Zanetti, A. Angellilo, V. Mattot, D. Nuyens, E. Lutgens, F. Clotman, M.C. de Ruiter, A. Gittenberger-de Groot, R. Poelmann, F. Lupu, J.M. Herbert, D. Collen, E. Dejana (1999) Targeted deficiency or cytosolic truncation of the VE-cadherin gene in mice impairs VEGF-mediated endothelial survival and angiogenesis. Cell 98: 147-157.

-Carnevale, E., E. Fogel, A.C. Aplin, M. Gelati, K.M. Howson, W.H. Zhu, R.F. Nicosia (2007) Regulation of postangiogenic neovessel survival by beta 1 and beta 3 integrins in collagen and fibrin matrices. J Vasc Res 44: 40-50.

Chan, A.C., S.G. Drakos, O.E. Ruiz, A.C. Smith, C.G. Gibson, J. Ling, S.F. Passi, A.N. Stratman, A. Sacharidou, M.P. Revelo, A.H. Grossmann, N.A. Diakos, G.E. Davis, M.M. Metzstein, K.J. Whitehead, D.Y. Li (2011) Loss of heterozygosity causes cerebral cavernous malformations in two distinct mouse models. J Clin Invest 121: 1871-1881.

Chang, S., B.D. Young, S. Li, X. Qi, J.A. Richardson, E.N. Olson (2006) Histone deacetylase 7 maintains vascular integrity by repressing matrix metalloproteinase 10. Cell 126: 321334.

Chen, T.T., A. Luque, S. Lee, S.M. Anderson, T. Segura, M.L. Iruela-Arispe (2010) Anchorage of VEGF to the extracellular matrix conveys differential signaling responses to endothelial cells. J Cell Biol 188: 595-609.

Chun, T.H., F. Sabeh, I. Ota, H. Murphy, K.T. McDonagh, K. Holmbeck, H. Birkedal-Hansen, E.D. Allen, S.J. Weiss (2004) MT1MMP-dependent neovessel formation within the confines of the three-dimensional extracellular matrix. J Cell Biol 167: 757-767.

Culver, J.C., M.E. Dickinson (2010) The effects of hemodynamic force on embryonic development. Microcirculation 17: 164-178.

Datta, A., D.M. Bryant, K.E. Mostov (2011) Molecular regulation of lumen morphogenesis. Curr Biol 21: R126-136.

Davis, G.E., K.J. Bayless (2003) An integrin and Rho GTPase-dependent pinocytic vacuole mechanism controls capillary lumen formation in collagen and fibrin matrices. Microcirculation 10: 27-44.

Davis, G.E., K.J. Bayless, A. Mavila (2002) Molecular basis of endothelial cell morphogenesis in three-dimensional extracellular matrices. Anat Rec 268: 252-275.
Davis, G.E., C.W. Camarillo (1996) An alpha 2 beta 1 integrin-dependent pinocytic mechanism involving intracellular vacuole formation and coalescence regulates capillary lumen and tube formation in three-dimensional collagen matrix. Exp Cell Res 224: 39-51.

Davis, G.E., W. Koh, A.N. Stratman (2007) Mechanisms controlling human endothelial lumen formation and tube assembly in three-dimensional extracellular matrices. Birth Defects Res C Embryo Today 81: 270 285.

Davis, G.E., W.B. Saunders (2006) Molecular balance of capillary tube formation versus regression in wound repair: role of matrix metalloproteinases and their inhibitors. J Investig Dermatol Symp Proc 11: 44-56.

Davis, G.E., D.R. Senger (2005) Endothelial extracellular matrix: biosynthesis, remodeling, and functions during vascular morphogenesis and neovessel stabilization. Circ Res 97: 1093-1107.

Davis, G.E., D.R. Senger (2008) Extracellular matrix mediates a molecular balance between vascular morphogenesis and regression. Curr Opin Hematol 15: 197-203.

Davis, G.E., A.N. Stratman, A. Sacharidou (2011a) Molecular control of vascular tube morphogenesis and stabilization: regulation by extracellular matrix, matrix metalloproteinases and endothelial cell-pericyte interactions; in Gerecht S. (ed): Biophysical Regulation of Vascular Differentiation. New York, Springer, pp 17-47.

Davis, G.E., A.N. Stratman, A. Sacharidou, W Koh (2011b) Molecular basis for endothelial lumen formation and tubulogenesis during vasculogenesis and angiogenic sprouting. Int Rev Cell Mol Biol 288: in press.

Dejana, E., E. Tournier-Lasserve, B.M. Weinstein (2009) The control of vascular integrity by endothelial cell junctions: molecular basis and pathological implications. Dev Cell 16: 209-221.

Dekan, G., A. Miettinen, E. Schnabel, M.G. Farquhar (1990) Binding of monoclonal antibodies to glomerular endothelium, slit membranes, and epithelium after in vivo injection: localization of antigens and bound IgGs by immunoelectron microscopy. Am J Pathol 137: 913-927.

Drake, C.J., L.A. Davis, C.D. Little (1992) Antibodies to beta 1-integrins cause alterations of aortic vasculogenesis, in vivo. Dev Dyn 193: 83-91.

Ebnet, K., M. Aurrand-Lions, A. Kuhn, F. Kiefer, S. Butz, K. Zander, M.K. Meyer zu Brickwedde, A. Suzuki, B.A. Imhof, D. Vestweber (2003) The junctional adhesion molecule (JAM) family members JAM-2 and JAM-3 associate with the cell polarity protein PAR3:a possible role for JAMs in endothelial cell polarity. J Cell Sci 116: 3879-3891. 
Ebnet, K., A. Suzuki, S. Ohno, D. Vestweber (2004) Junctional adhesion molecules (JAMs): more molecules with dual functions? J Cell Sci 117: 19-29.

Egginton, S., M. Gerritsen (2003) Lumen formation: in vivo versus in vitro observations. $\mathrm{Mi}$ crocirculation 10: 45-61.

Filla, M.B., A. Czirok, E.A. Zamir, C.D. Little, T.J. Cheuvront, B.J. Rongish (2004) Dynamic imaging of cell, extracellular matrix, and tissue movements during avian vertebral axis patterning. Birth Defects Res C Embryo Today 72: 267-276.

-Fisher, K.E., A. Sacharidou, A.N. Stratman, A.M. Mayo, S.B. Fisher, R.D. Mahan, M.J. Davis, G.E. Davis (2009) MT1-MMP- and Cdc42dependent signaling co-regulate cell invasion and tunnel formation in 3D collagen matrices. J Cell Sci 122: 4558-4569.

Folkman, J., C. Haudenschild (1980) Angiogenesis in vitro. Nature 288: 551-556.

Francis, S.E., K.L. Goh, K. Hodivala-Dilke, B.L. Bader, M. Stark, D. Davidson, R.O. Hynes (2002) Central roles of alpha5beta1 integrin and fibronectin in vascular development in mouse embryos and embryoid bodies. Arterioscler Thromb Vasc Biol 22: 927-933.

-Gaengel, K., G. Genove, A. Armulik, C. Betsholtz (2009) Endothelial-mural cell signaling in vascular development and angiogenesis. Arterioscler Thromb Vasc Biol 29: 630-638.

Galan Moya, E.M., A. Le Guelte, J. Gavard (2009) PAKing up to the endothelium. Cell Signal 21: 1727-1737.

Glading, A., J. Han, R.A. Stockton, M.H. Ginsberg (2007) KRIT-1/CCM1 is a Rap1 effector that regulates endothelial cell cell junctions. J Cell Biol 179: 247-254.

Hao, Y., Q. Du, X. Chen, Z. Zheng, J.L. Balsbaugh, S. Maitra, J. Shabanowitz, D.F. Hunt, I.G. Macara (2010) Par3 controls epithelial spindle orientation by aPKC-mediated phosphorylation of apical pins. Curr Biol 20: 1809-1818.

Herbert, S.P., J. Huisken, T.N. Kim, M.E. Feldman, B.T. Houseman, R.A. Wang, K.M. Shokat, D.Y. Stainier (2009) Arterial-venous segregation by selective cell sprouting: an alternative mode of blood vessel formation. Science 326: 294-298.

-Hill, M.A., Z. Sun, L. Martinez-Lemus, G.A. Meininger (2007) New technologies for dissecting the arteriolar myogenic response. Trends Pharmacol Sci 28: 308-315.

Hoang, M.V., J.A. Nagy, D.R. Senger (2010a) Active Racl improves pathological VEGF neovessel architecture and reduces vascular leak: mechanistic similarities with angiopoietin-1. Blood 117: 1751-1760.

Hoang, M.V., J.A. Nagy, D.R. Senger (2010b) Cdc42-mediated inhibition of GSK-3beta improves angio-architecture and lumen formation during VEGF-driven pathological angiogenesis. Microvasc Res 81: 34-43.
Holderfield, M.T., C.C. Hughes (2008) Crosstalk between vascular endothelial growth factor, notch, and transforming growth factor-beta in vascular morphogenesis. Circ Res 102: 637-652.

Hughes, C.C. (2008) Endothelial-stromal interactions in angiogenesis. Curr Opin Hematol 15: 204-209.

Hynes, R.O. (2007) Cell-matrix adhesion in vascular development. J Thromb Haemost 5(suppl 1): 32-40.

Hynes, R.O. (2009) The extracellular matrix: not just pretty fibrils. Science 326: 1216-1219.

Im, E., A. Kazlauskas (2007) Src family kinases promote vessel stability by antagonizing the Rho/ROCK pathway. J Biol Chem 282: 29122-29129.

-Iruela-Arispe, M.L., G.E. Davis (2009) Cellular and molecular mechanisms of vascular lumen formation. Dev Cell 16: 222-231.

-Jaffe, A.B., N. Kaji, J. Durgan, A. Hall (2008) Cdc42 controls spindle orientation to position the apical surface during epithelial morphogenesis. J Cell Biol 183: 625-633.

Kamei, M., W.B. Saunders, K.J. Bayless, L. Dye, G.E. Davis, B.M. Weinstein (2006) Endothelial tubes assemble from intracellular vacuoles in vivo. Nature 442: 453-456.

Kiosses, W.B., J. Hood, S. Yang, M.E. Gerritsen, D.A. Cheresh, N. Alderson, M.A. Schwartz (2002) A dominant-negative p65 PAK peptide inhibits angiogenesis. Circ Res 90: 697702.

Kleaveland, B., X. Zheng, J.J. Liu, Y. Blum, J.J. Tung, Z. Zou, S.M. Sweeney, M. Chen, L. Guo, M.M. Lu, D. Zhou, J. Kitajewski, M. Affolter, M.H. Ginsberg, M.L. Kahn (2009) Regulation of cardiovascular development and integrity by the heart of glass-cerebral cavernous malformation protein pathway. Nat Med 15: 169-176.

Koh, W., R.D. Mahan, G.E. Davis (2008a) Cdc42and Racl-mediated endothelial lumen formation requires Pak2, Pak4 and Par3, and PKC-dependent signaling. J Cell Sci 121: 989-1001.

Koh, W., K. Sachidanandam, A.N. Stratman, A. Sacharidou, A.M. Mayo, E.A. Murphy, D.A. Cheresh, G.E. Davis (2009) Formation of endothelial lumens requires a coordinated PKCepsilon-, Src-, Pak- and Raf-kinase-dependent signaling cascade downstream of Cdc42 activation. J Cell Sci 122: 1812-1822.

Koh, W., A.N. Stratman, A. Sacharidou, G.E. Davis (2008b) In vitro three dimensional collagen matrix models of endothelial lumen formation during vasculogenesis and angiogenesis. Methods Enzymol 443: 83-101.

Lafleur, M.A., M.M. Handsley, V. Knauper, G. Murphy, D.R. Edwards (2002) Endothelial tubulogenesis within fibrin gels specifically requires the activity of membrane-type-matrix metalloproteinases (MT-MMPs). J Cell Sci 115: 3427-3438.
Lamagna, C., K.M. Hodivala-Dilke, B.A. Imhof, M. Aurrand-Lions (2005) Antibody against junctional adhesion molecule-C inhibits angiogenesis and tumor growth. Cancer Res 65: 5703-5710.

Lampugnani, M.G., E. Dejana (2007) Adherens junctions in endothelial cells regulate vessel maintenance and angiogenesis. Thromb Res 120(suppl 2): S1-6.

Lampugnani, M.G., F. Orsenigo, N. Rudini, L. Maddaluno, G. Boulday, F. Chapon, E. Dejana (2010) CCM1 regulates vascular-lumen organization by inducing endothelial polarity. J Cell Sci 123: 1073-1080.

Liu, J., S.D. Fraser, P.W. Faloon, E.L. Rollins, J. Vom Berg, O. Starovic-Subota, A.L. Laliberte, J.N. Chen, F.C. Serluca, S.J. Childs (2007) A betaPix Pak2a signaling pathway regulates cerebral vascular stability in zebrafish. Proc Natl Acad Sci USA 104: 13990 13995.

Liu, H., D. Rigamonti, A. Badr, J. Zhang (2010) $\mathrm{Ccm} 1$ regulates microvascular morphogenesis during angiogenesis. J Vasc Res 48: 130140.

Liu, Y., D.R. Senger (2004) Matrix-specific activation of Src and Rho initiates capillary morphogenesis of endothelial cells. FASEB J 18: 457-468.

Lubarsky, B., M.A. Krasnow (2003) Tube morphogenesis: making and shaping biological tubes. Cell 112: 19-28.

Lucitti, J.L., E.A. Jones, C. Huang, J. Chen, S.E. Fraser, M.E. Dickinson (2007) Vascular remodeling of the mouse yolk sac requires hemodynamic force. Development 134: 33173326.

Martin-Belmonte, F., A. Gassama, A. Datta, W. Yu, U. Rescher, V. Gerke, K. Mostov (2007) PTEN-mediated apical segregation of phosphoinositides controls epithelial morphogenesis through Cdc42. Cell 128: 383-397.

Martin-Belmonte, F., W. Yu, A.E. RodriguezFraticelli, A.J. Ewald, Z. Werb, M.A. Alonso, K. Mostov (2008) Cell-polarity dynamics controls the mechanism of lumen formation in epithelial morphogenesis. Curr Biol 18: 507-513.

Mavria, G., Y. Vercoulen, M. Yeo, H. Paterson, M. Karasarides, R. Marais, D. Bird, C.J. Marshall (2006) ERK-MAPK signaling opposes Rho-kinase to promote endothelial cell survival and sprouting during angiogenesis. Cancer Cell 9: 33-44.

McKinney, M.C., B.M. Weinstein (2008) Chapter 4: using the zebrafish to study vessel formation. Methods Enzymol 444: 65-97.

Mochida, G.H., V.S. Ganesh, J.M. Felie, D. Gleason, R.S. Hill, K.R. Clapham, D. Rakiec, W.H. Tan, N. Akawi, M. Al-Saffar, J.N. Partlow, S. Tinschert, A.J. Barkovich, B. Ali, L. Al-Gazali, C.A. Walsh (2011) A homozygous mutation in the tight-junction protein JAM3 causes hemorrhagic destruction of the brain, subependymal calcification, and congenital cataracts. Am J Hum Genet 87: 882-889. 
Nakatsu, M.N., C.C. Hughes (2008) An optimized three-dimensional in vitro model for the analysis of angiogenesis. Methods Enzymol 443: 65-82.

O’Brien, L.E., W. Yu, K. Tang, T.S. Jou, M.M. Zegers, K.E. Mostov (2006) Morphological and biochemical analysis of Racl in threedimensional epithelial cell cultures. Methods Enzymol 406: 676-691.

Rhodes, J.M., M. Simons (2007) The extracellular matrix and blood vessel formation: not just a scaffold. J Cell Mol Med 11: 176-205.

Sabeh, F., R. Shimizu-Hirota, S.J. Weiss (2009) Protease-dependent versus -independent cancer cell invasion programs: three-dimensional amoeboid movement revisited. J Cell Biol 185: 11-19.

- Sacharidou, A., W. Koh, A.N. Stratman, A.M. Mayo, K.E. Fisher, G.E. Davis (2010) Endothelial lumen signaling complexes control 3D matrix-specific tubulogenesis through interdependent Cdc42- and MT1-MMP-mediated events. Blood 115: 5259-5269.

-Sainson, R.C., J. Aoto, M.N. Nakatsu, M. Holderfield, E. Conn, E. Koller, C.C. Hughes (2005) Cell-autonomous notch signaling regulates endothelial cell branching and proliferation during vascular tubulogenesis. FASEB J 19: 1027-1029.

-San Antonio, J.D., J.J. Zoeller, K. Habursky, K. Turner, W. Pimtong, M. Burrows, S. Choi, S. Basra, J.S. Bennett, W.F. DeGrado, R.V. Iozzo (2009) A key role for the integrin alpha2beta1 in experimental and developmental angiogenesis. Am J Pathol 175: 1338-1347.

-Sato, Y., G. Poynter, D. Huss, M.B. Filla, A. Czirok, B.J. Rongish, C.D. Little, S.E. Fraser, R. Lansford (2010) Dynamic analysis of vascular morphogenesis using transgenic quail embryos. PLoS One 5: e12674.

-Saunders, W.B., K.J. Bayless, G.E. Davis (2005) MMP-1 activation by serine proteases and MMP-10 induces human capillary tubular network collapse and regression in 3D collagen matrices. J Cell Sci 118: 2325-2340.

-Saunders, W.B., B.L. Bohnsack, J.B. Faske, N.J. Anthis, K.J. Bayless, K.K. Hirschi, G.E. Davis (2006) Coregulation of vascular tube stabilization by endothelial cell TIMP-2 and pericyte TIMP-3. J Cell Biol 175: 179-191.

-Segal, S.S. (2005) Regulation of blood flow in the microcirculation. Microcirculation 12: 3345.

-Senger, D.R., K.P. Claffey, J.E. Benes, C.A. Perruzzi, A.P. Sergiou, M. Detmar (1997) Angiogenesis promoted by vascular endothelial growth factor: regulation through alphalbeta1 and alpha2betal integrins. Proc Natl Acad Sci USA 94: 13612-13617.

-Senger, D.R., G.E. Davis (2011) Angiogenesis. Cold Spring Harb Perspect Biol 3: a005090.
Somanath, P.R., A. Ciocea, T.V. Byzova (2009) Integrin and growth factor receptor alliance in angiogenesis. Cell Biochem Biophys 53: $53-64$.

Stockton, R.A., R. Shenkar, I.A. Awad, M.H. Ginsberg (2010) Cerebral cavernous malformations proteins inhibit Rho kinase to stabilize vascular integrity. J Exp Med 207: 881896.

Stratman, A.N., M.J. Davis, G.E. Davis (2011) VEGF and FGF prime vascular tube morphogenesis and sprouting directed by hematopoietic stem cell cytokines. Blood 117: 3709-3719.

Stratman, A.N., K.M. Malotte, R.D. Mahan, M.J. Davis, G.E. Davis (2009a) Pericyte recruitment during vasculogenic tube assembly stimulates endothelial basement membrane matrix formation. Blood 114: 5091-5101.

- Stratman, A.N., W.B. Saunders, A. Sacharidou, W. Koh, K.E. Fisher, D.C. Zawieja, M.J. Davis, G.E. Davis (2009b) Endothelial cell lumen and vascular guidance tunnel formation requires MT1-MMP-dependent proteolysis in 3-dimensional collagen matrices. Blood 114: 237-247.

Stratman, A.N., A.E. Schwindt, K.M. Malotte, G.E. Davis (2010) Endothelial-derived PDGF-BB and HB-EGF coordinately regulate pericyte recruitment during vasculogenic tube assembly and stabilization. Blood 116: $4720-4730$.

Strilic, B., T. Kucera, J. Eglinger, M.R. Hughes, K.M. McNagny, S. Tsukita, E. Dejana, N. Ferrara, E. Lammert (2009) The molecular basis of vascular lumen formation in the developing mouse aorta. Dev Cell 17: 505-515.

Stupack, D.G., D.A. Cheresh (2004) Integrins and angiogenesis. Curr Top Dev Biol 64: 207-238.

Tan, W., T.R. Palmby, J. Gavard, P. Amornphimoltham, Y. Zheng, J.S. Gutkind (2008) An essential role for Racl in endothelial cell function and vascular development. FASEB J 22: 1829-1838.

Tian, Y., L. Lei, M. Cammarano, T. Nekrasova, A. Minden (2009) Essential role for the Pak4 protein kinase in extraembryonic tissue development and vessel formation. Mech Dev 126: 710-720.

Tzima, E., W.B. Kiosses, M.A. del Pozo, M.A. Schwartz (2003) Localized cdc42 activation, detected using a novel assay, mediates microtubule organizing center positioning in endothelial cells in response to fluid shear stress. J Biol Chem 278: 31020-31023.

van der Flier, A., K. Badu-Nkansah, C.A. Whittaker, D. Crowley, R.T. Bronson, A. LacyHulbert, R.O. Hynes (2010) Endothelial alpha5 and alphav integrins cooperate in remodeling of the vasculature during development. Development 137: 2439-2449.
Wagenseil, J.E., R.P. Mecham (2009) Vascular extracellular matrix and arterial mechanics. Physiol Rev 89: 957-989.

Wang, Y., M.S. Kaiser, J.D. Larson, A. Nasevicius, K.J. Clark, S.A. Wadman, S.E. RobergPerez, S.C. Ekker, P.B. Hackett, M. McGrail, J.J. Essner (2010) Moesin1 and Ve-cadherin are required in endothelial cells during in vivo tubulogenesis. Development 137: 31193128.

Warren, C.M., M.L. Iruela-Arispe (2010) Signaling circuitry in vascular morphogenesis. Curr Opin Hematol 17: 213-218.

Whitehead, K.J., A.C. Chan, S. Navankasattusas, W. Koh, N.R. London, J. Ling, A.H. Mayo, S.G. Drakos, C.A. Jones, W. Zhu, D.A. Marchuk, G.E. Davis, D.Y. Li (2009) The cerebral cavernous malformation signaling pathway promotes vascular integrity via Rho GTPases. Nat Med 15: 177-184.

Xu, K., D.C. Chong, S.A. Rankin, A.M. Zorn, O. Cleaver (2009) Rasip1 is required for endothelial cell motility, angiogenesis and vessel formation. Dev Biol 329: 269-279.

Xu, K., A. Sacharidou, S. Fu, D.C. Chong, B. Skaug, Z.J. Chen, G.E. Davis, O. Cleaver (2011) Blood vessel tubulogenesis requires Rasip1 regulation of GTPase signaling. Dev Cell 20: 1-14.

Yang, S., J. Graham, J.W. Kahn, E.A. Schwartz, M.E. Gerritsen (1999) Functional roles for PECAM-1 (CD31) and VE-cadherin (CD144) in tube assembly and lumen formation in three-dimensional collagen gels. Am J Pathol 155: 887-895.

-Yaniv, K., S. Isogai, D. Castranova, L. Dye, J. Hitomi, B.M. Weinstein (2006) Live imaging of lymphatic development in the zebrafish. Nat Med 12: 711-716.

Zamir, E.A., A. Czirok, C. Cui, C.D. Little, B.J. Rongish (2006) Mesodermal cell displacements during avian gastrulation are due to both individual cell-autonomous and convective tissue movements. Proc Natl Acad Sci USA 103: 19806-19811.

Zheng, X., C. Xu, A. Di Lorenzo, B. Kleaveland, Z. Zou, C. Seiler, M. Chen, L. Cheng, J. Xiao, J. He, M.A. Pack, W.C. Sessa, M.L. Kahn (2010) CCM3 signaling through sterile 20like kinases plays an essential role during zebrafish cardiovascular development and cerebral cavernous malformations. J Clin Invest 120: 2795-2804.

Zovein, A.C., A. Luque, K.A. Turlo, J.J. Hofmann, K.M. Yee, M.S. Becker, R. Fassler, I. Mellman, T.F. Lane, M.L. Iruela-Arispe (2010) $\beta 1$ integrin establishes endothelial cell polarity and arteriolar lumen formation via a Par3-dependent mechanism. Dev Cell 18: 39-51. 